\title{
Synthetic studies related to the akuammiline alkaloids
}

\author{
Carme Cantos Llopart and John A. Joule \\ Chemistry Department, The University of Manchester, Manchester M13 9PL, U.K. \\ E-mail: j.a.joule@man.ac.uk \\ Dedicated to Professor Rod Rickards on his $70^{\text {th }}$ birthday \\ (received 07 Jan 04; accepted 15 Apr 04; published on the web 19 Apr 04)

\begin{abstract}
6-Ethyl-2,3-dihydro-1-phenyl-1H-pyrrolizine 19 and 1-(2-aminophenyl)-6-ethyl-2,3-dihydro$1 H$-pyrrolizine 30 were synthesized starting from the pyrroles 4-acetylpyrrol-2-yl phenyl ketone 13 and 4-acetylpyrrol-2-yl 2-(dimethylaminomethylenamino)phenyl ketone 26, respectively, using vinyltriphenylphosphonium bromide in an intramolecular Wittig reaction for the formation of the second five-membered ring.
\end{abstract}

Keywords: Pyrroles, pyrrolizidines, akuamma alkaloids, vinyltriphenylphosphonium bromide, Wittig reaction

\section{Introduction}

The variety of indole alkaloid skeleta, ${ }^{1}$ of greater or lesser complexity, has been a frequently employed proving ground for novel synthetic methodologies and strategies. Some indole alkaloid types have been the subject of frequent attention - the alkaloid ellipticine ${ }^{2}$ probably holds the record for the most frequently synthesised alkaloid ever - the Aspidosperma skeleton ${ }^{3}$ has seen Stork's synthesis, an early illustration of enamine $\beta$-alkylation, Overman's approach, illustrating his aza-Cope/Mannich sequence, and Magnus' route utilising an indole-2,3-quinodimethane intermediate, amongst others. One of the few groups of monoterpenoid indole alkaloids which has not yet been the subject of a successful total synthesis, is the group characterised by the presence of a C-7-C-16 bond $^{4}$ - akuammiline 1 represents this structural type in its simplest form. Approximately 100 indole bases are now known (more than 20 in the last decade) which fall into the akuammiline structural category, and the chemistry and pharmacology of these have been recently reviewed. ${ }^{5}$

Our personal interest in this group of alkaloids traces back to that period when the Manchester Chemistry Department was priviliged to number amongst its academic staff Arthur Birch and Rod Rickards, and one of the present authors studied Akuamma alkaloids for PhD. ${ }^{6}$ It is a pleasure to contribute this paper for the issue of Arkivoc which commemorates Professor 
Rickards' 70th Birthday, in recognition of his contributions to natural product chemistry in Manchester, and subsequently in Canberra, and of his friendship and encouragement as a colleague in Manchester.

\section{Background}

The use of plants and plant extracts containing akuammiline alkaloids is widespread in traditional medicine. For example, the aqueous extracts of Picralima nitida (the "akuamma” tree) are used in West Africa as painkillers; ${ }^{7}$ in Ghana, pulverised and encapsulated seeds can be found for sale for medicinal purposes in the market. In the rural areas of Saudi Arabia, decoctions of Rhazya stricta are employed as a cure for helminthiasis ${ }^{8}$ while in the Philippines the bark of Rauwolfia sumatrana is believed to alleviate the symptoms of malaria. ${ }^{9}$

Prompted by the traditional medicinal use of the seeds of the Picralima nitida tree in the Gold Coast, Henry and Sharp were the first to examine the alkaloidal content. ${ }^{10}$ These authors adopted the word "akuamma", used by natives in the region to designate the tree, as the prefix for the name given to the major alkaloidal constituent, akuammine 2 ( $\mathrm{R}=\mathrm{HO}$ ). Subsequently, Henry isolated, and characterised $\Psi$-akuammigine $2(\mathrm{R}=\mathrm{H})$ and akuammiline. ${ }^{11}$ In 1961 , the determination of the X-ray crystal structure of the quaternary salt echitamine $3^{12}$ then allowed the final establishment of the structures and absolute configurations ${ }^{13}$ of $\Psi$-akuammigine, akuammine and akuammiline based on their chemical correlation with echitamine.

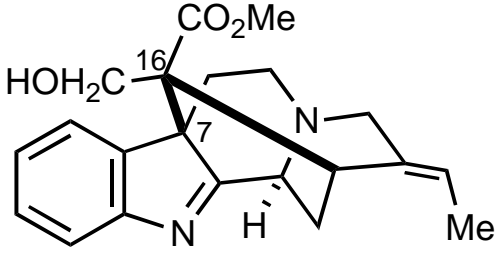

1

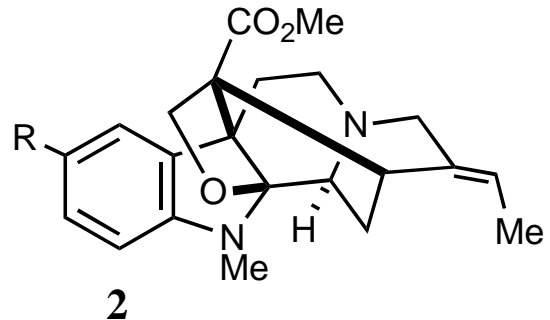

2

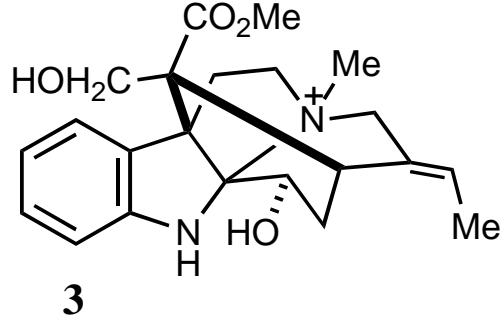

3
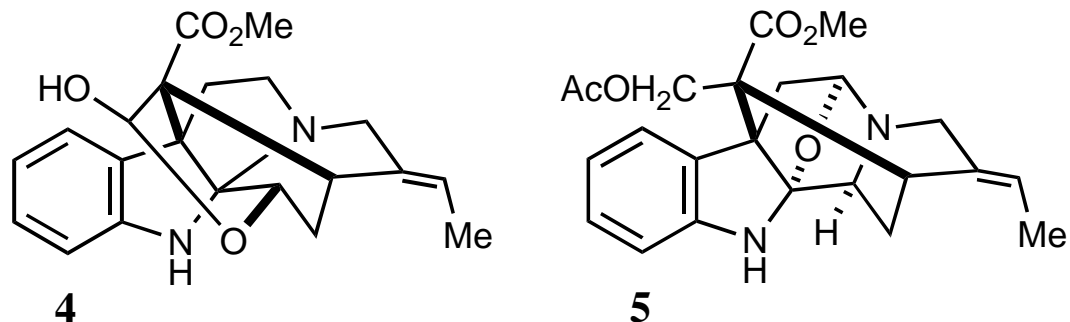

5

Studies of extracts of seeds, fruit and bark of Picralima nitida revealed anti-protozoal, antimicrobial and in vitro anti-malarial activity. ${ }^{14}$ Extracts from Hunteria zeylanica (rich in corymine 4) showed anti-inflammatory activity and stimulation of the CNS, ${ }^{15}$ while extracts from Alstonia scholaris (which contain picraline $\mathbf{5}$ and $\Psi$-akuammigine amongst other akuammiline alkaloids) showed hepato-protective activity. ${ }^{16}$ Echitamine chloride has been reported to have antitumour activity coupled with a low toxicity profile. ${ }^{17}$ It is probably appropriate to apply the term privileged structures, as employed by Evans et al. ${ }^{18}$ to designate 
those substrates that bind to diverse categories of protein receptors with high affinities, to the unique rigid skeleton of the Akuamma alkaloids, exposing as it does, a variety of functional groups in a defined alignment.

\section{Previous synthetic approaches}

Early work ${ }^{19,20}$ was followed by attempts to achieve formation of a C-7-C-16 bond by a means parallel to that proposed ${ }^{21}$ for its biosynthetic formation, but these were unsuccessful. ${ }^{22}$ Lévy $^{23}$ described a significantly novel approach, which awaits further development, but the most extensive and interesting, though also, in the end, unsuccessful work has come from the Catalan group of Bosch and Bennasar. ${ }^{24}$

It remains the situation that no successful synthesis of the Akuamma skeleton has been achieved; this paper describes work that is designed to provide the platform for an alternative strategy for the synthesis of this type of indole alkaloid.

\section{Synthetic plan}

The route proposed for the assembly of molecules with the akuammiline skeleton is summarised in retrosynthetic Scheme 1. A key step would be an aza-Diels-Alder cycloaddition to form ring D (7 to 6). Scheme 1 shows this as an intermolecular process, though an alternative would be to bring this about in an intramolecular sense, with the acrylate linked to $\mathrm{N}-1$, i.e. with the dienophile as an acrylamide. We envisage producing the required 1-aza-1,3-diene 7 by an oxidative ring opening of a precursor bicyclic pyrrole $\mathbf{8}$. The conversion of the bicyclic pyrrole into the azadiene would be based on one of two precedents: (1) the first is the addition ${ }^{25}$ of singlet oxygen to a pyrrole, eventually producing a 5-hydroxy-5H-pyrrol-2-one, ${ }^{26}$ which, we speculate, would react with TMSOTf to produce a ring-cleaved azadiene-ketone ready for cycloaddition; (2) alternatively, based on Martin's work in a furan series, ${ }^{27}$ the use of tert$\mathrm{BuOOH} / \mathrm{VO}(\mathrm{acac})_{2}$, on a pyrrol-2-ylcarbinol might have a comparable effect. In this paper we describe our synthesis of an appropriate bicyclic pyrrole, $\mathbf{8}$.

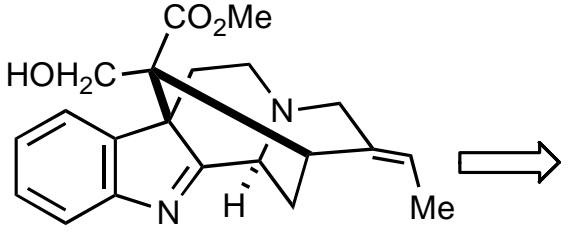

1
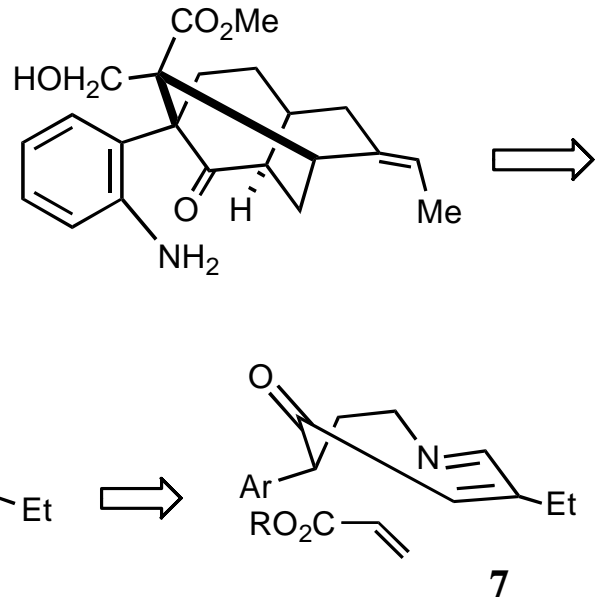
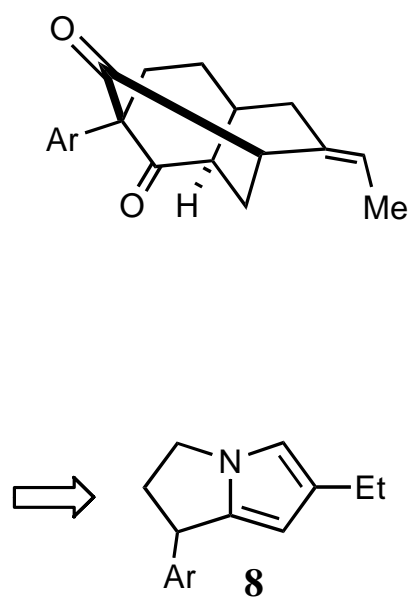

Scheme 1 


\section{Synthesis of bicycles 8}

Our first plan (Scheme 2) was to close an intermediate of the form 9, via an intramolecular electrophilic attack at the pyrrole $\alpha$-position (ignoring questions of regioselectivity), to give $\mathbf{1 0}$. 2-Aminoethyl phenyl ketone hydrochloride ${ }^{28}$ was prepared via addition of phthalimide to phenyl vinyl ketone, ${ }^{29}$ then hydrolysis with 1:1 AcOH-conc $\mathrm{HCl}^{30}$ Reaction of the amine with 2,5dimethoxytetrahydrofuran gave the pyrrole ketone 9a, borohydride reduction of which gave the alcohol 9b. Alcohol 9b was also prepared from 3-hydroxy-3-phenylpropanamine ${ }^{31}$ by reaction ${ }^{32}$ with 2,5-dimethoxytetrahydrofuran.

Attempts to effect ring closure by conversion of the benzylic alcohol in $\mathbf{9 b}$ into a triflate were disappointing. Despite seemingly excellent precedents in which an analogous 5,6-ring system was constructed, ${ }^{33}$ the best that could be claimed in our experiments were signals in ${ }^{1} \mathrm{H}$ NMR spectra, of complex product mixtures, which could have represented some of the desired product. We explored the possibility of achieving the desired benzylic electrophilic reactivity by decomposition of a trichloroacetimidate; ${ }^{34,35}$ the required derivative $9 \mathbf{c}$ was easily prepared following a literature precedent, ${ }^{36}$ but various attempts to effect the desired acid-catalysed closure were unsuccessful (Scheme 2).
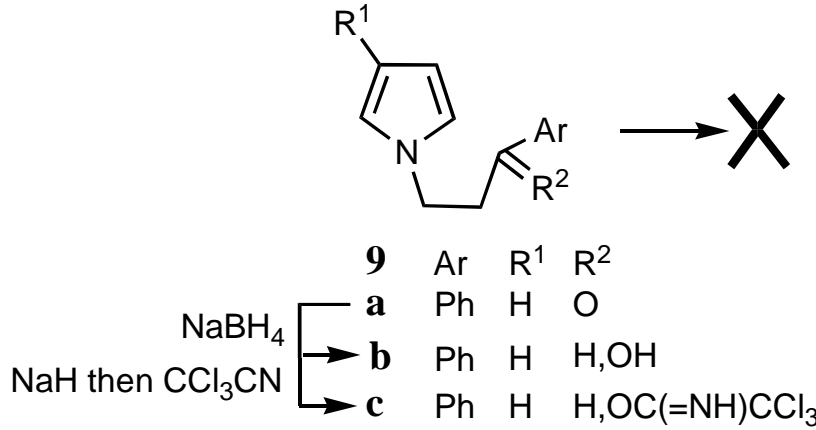

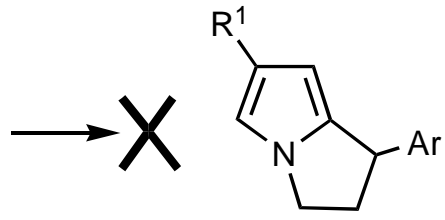

10

\section{Scheme 2}

Katritzky had described ${ }^{37}$ the synthesis of bicycles 12a and 12b, the key step, in which the saturated five-membered ring is fused to the pyrrole nucleus, involving intramolecular displacement of tosylate in 2-(benzotriazol-1-ylmethyl)-1-(2-tosyloxyethyl)pyrrole precursors 11a and 11b, subsequent nucleophilic displacement of the benzotriazolyl unit in 12a with phenylmagnesium bromide completing a synthesis of 13a. Although we were able to reproduce the literature report and prepare 13a, in attempting to adapt this sequence for the synthesis of the desired 13b, we were only able to reach 11c, as shown in Scheme 3, failing completely in attempts to bring about ring closure to 12c from this intermediate.

We turned to construction of the five-membered ring via an intramolecular Wittig reaction. Linderman and Meyers had $\operatorname{shown}^{38}$ that addition of pyrrolyl anions to vinyltriphenylphosphonium bromide, directly generates ylides which react with aldehydes to produce substituted 1-allylpyrroles. The process had been earlier carried out in an intramolecular sense starting from 2-formylpyrrole, to generate a five-five bicycle of the type required in the 
present work, though an attempt to use 2-benzoylpyrrole produced only tars. ${ }^{39}$ A comparable five-membered ring formation was reported with 2-formylindole. ${ }^{39}$

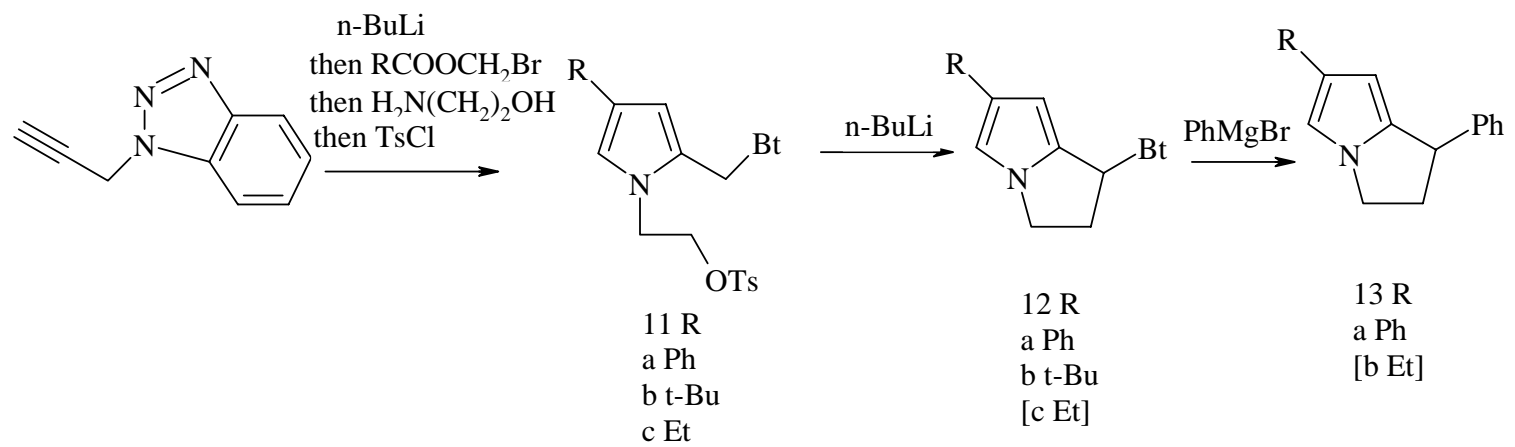

\section{Scheme 3}

In our hands, a preliminary investigation using 2-benzoylpyrrole in reaction with vinyltriphenylphosphonium bromide was encouraging but sounded a warning: there was clear evidence that an initial product 14 was in equilibrium (ca. 1:1) with an isomer $\mathbf{1 5}$ (Scheme 4). In an attempt to prevent this complication, we turned to the use of a 3-acetylpyrrole, arguing that conjugation with this ketone carbonyl would maintain the first-formed system of double bonds. The acetyl substituent also corresponds to the two-carbon side-chain eventually required in the alkaloid.

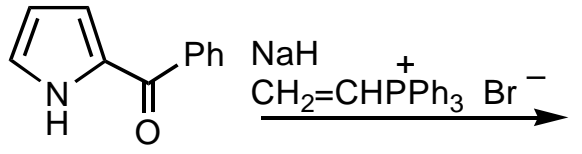<smiles>C1=C(c2ccccc2)c2cccn2C1</smiles>

14

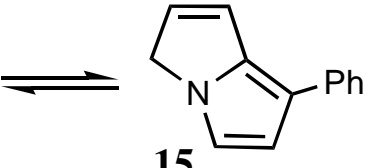

15

\section{Scheme 4}

Of various routes to 4-acetyl-2-benzoylpyrrole $\mathbf{1 6}$ we found the best was to start with 3acetylpyrrole, itself available via Friedel-Crafts acetylation of 1-phenylsulfonylpyrrole, then de$N$-protection; ${ }^{40,41}$ benzoylation of 3-acetylpyrrole gave 16 in $20 \%$ overall yield for the four steps from pyrrole.
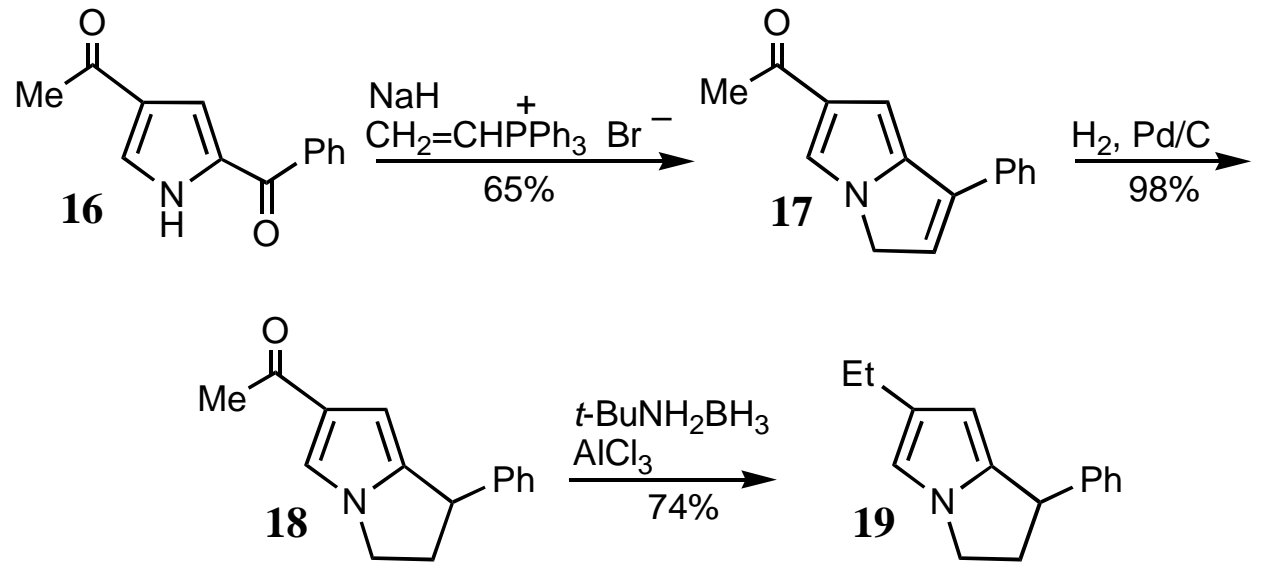

\section{Scheme 5}


Reaction of 16 with vinyltriphenylphosphonium bromide produced a single, though rather unstable bicyclic alkene 17, which was hydrogenated giving 18, the acetyl group then reduced, producing 19, using ${ }^{42} t-\mathrm{BuNH}_{2} \mathrm{BH}_{3}$ with $\mathrm{AlCl}_{3}$ (Scheme 5). That the orientation of double bonds was, as desired, and as shown in 17, 18 and 19, was verified by the ${ }^{1} \mathrm{H}$ NMR spectra of these substances. For example, in 17 the methylene signal at $\delta 4.68$ was split ( $J 2.3 \mathrm{~Hz}$ ) by the adjacent alkene proton and in the spectrum of 18 there was a triplet $(J 7.7)$ at $\delta 4.40$ for the doubly benzylic proton, in addition to multiplet signals for the other four aliphatic protons.

The further development of this successful route, to provide an appropriate bicycle for the alkaloid synthesis, required that the benzenoid ring carry an ortho nitrogen substitutent. We reacted the 2-lithio-1-phenylsulfonylpyrrole with ortho-nitrobenzaldehyde, then immediately oxidised the resulting mixture, but were only able to obtain a 10\% yield of ketone $\mathbf{2 0}$. However, following work using 1-para-toluenesulfonylpyrrole, ${ }^{43}$ Friedel-Crafts ortho-nitrobenzoylation of 1-phenylsulfonylpyrrole proceeded satisfactorily, giving 20, the orientation of substitution being confirmed by the ${ }^{1} \mathrm{H}-\mathrm{NMR}$ spectrum of the product which showed signals for the three orthorelated pyrrole protons at $\delta 6.34(1 \mathrm{H}, \mathrm{m}), 6.50(1 \mathrm{H}, \mathrm{dd}, J 1.8,3.8 \mathrm{~Hz})$ and $7.97(1 \mathrm{H}, \mathrm{dd}, J 1.8,3.8$ $\mathrm{Hz}$ ). After $\mathrm{N}$-deprotection, $\mathrm{AlCl}_{3}$-catalysed acetylation gave the desired diketone 21, by substitution meta to the nitrobenzoyl group, as confirmed by the small coupling constants for the remaining two pyrrole protons, at $\delta 6.90$ (bs) and 7.92 (bs). Disappointingly, the Wittig cyclisation with nitro-substituted substrate $\mathbf{2 1}$ failed completely - only polar material that could not be characterised was obtained (Scheme 6).
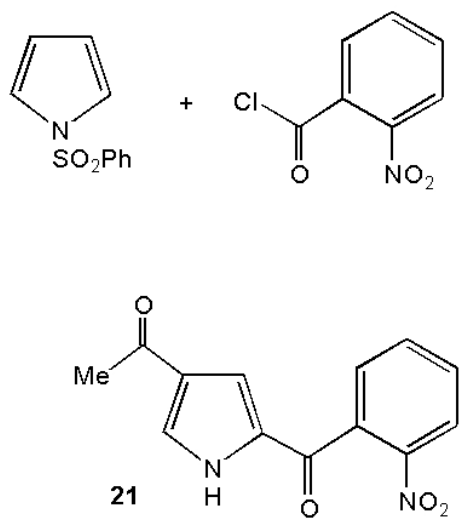

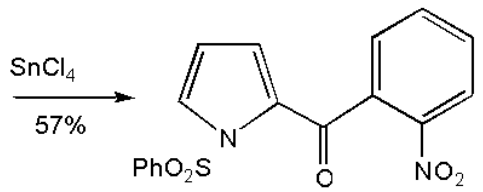

20
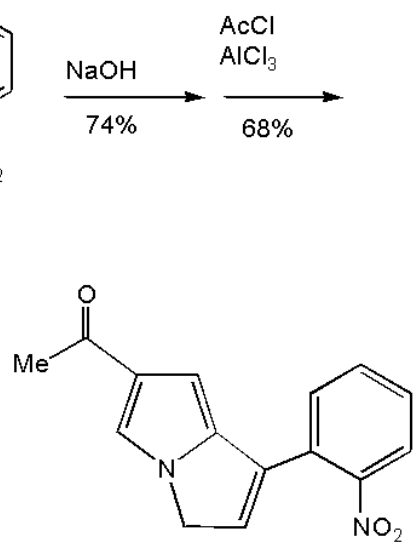

\section{Scheme 6}

It was clear that an alternative nitrogen substitutent would have to be used - a protected amine was the obvious choice. There appeared to be a rather direct route through to a benzamide following a strong earlier precedent. ${ }^{44}$ Anthranilic acid was converted into 2-phenyl-4Hbenzoxazin-4-one 22 by reaction with benzoyl chloride and this reacted with the Grignard derivative of pyrrole giving 23. C-Acetylation proceeded normally and the resulting diketone 24a was subjected to the Wittig cyclisation conditions, but once again we were to be disappointed, and no bicyclic product was obtained (Scheme 7). There are two $N$-hydrogens in 
24a and the cyclisation requires that base remove the pyrrole $N$-hydrogen. By reaction of 24a with one equivalent of $\mathrm{NaH}$ and then iodomethane we confirmed that the pyrrole $N$-proton is the most acidic proton, by the formation of $\mathbf{2 4 b}$, in which the typical signal for a pyrrole $N$-hydrogen ( $\delta 10.6$ in 24a) was no longer present, but the amide proton signal remained, and at exactly the same shift, $\delta 11.4$, as in 24a. It is not clear why 24a would not take part in the Wittig cyclisation. Hydrolysis of the benzamide revealed the amine 25, but, as expected, no Wittig cyclisation could be achieved with this, either.
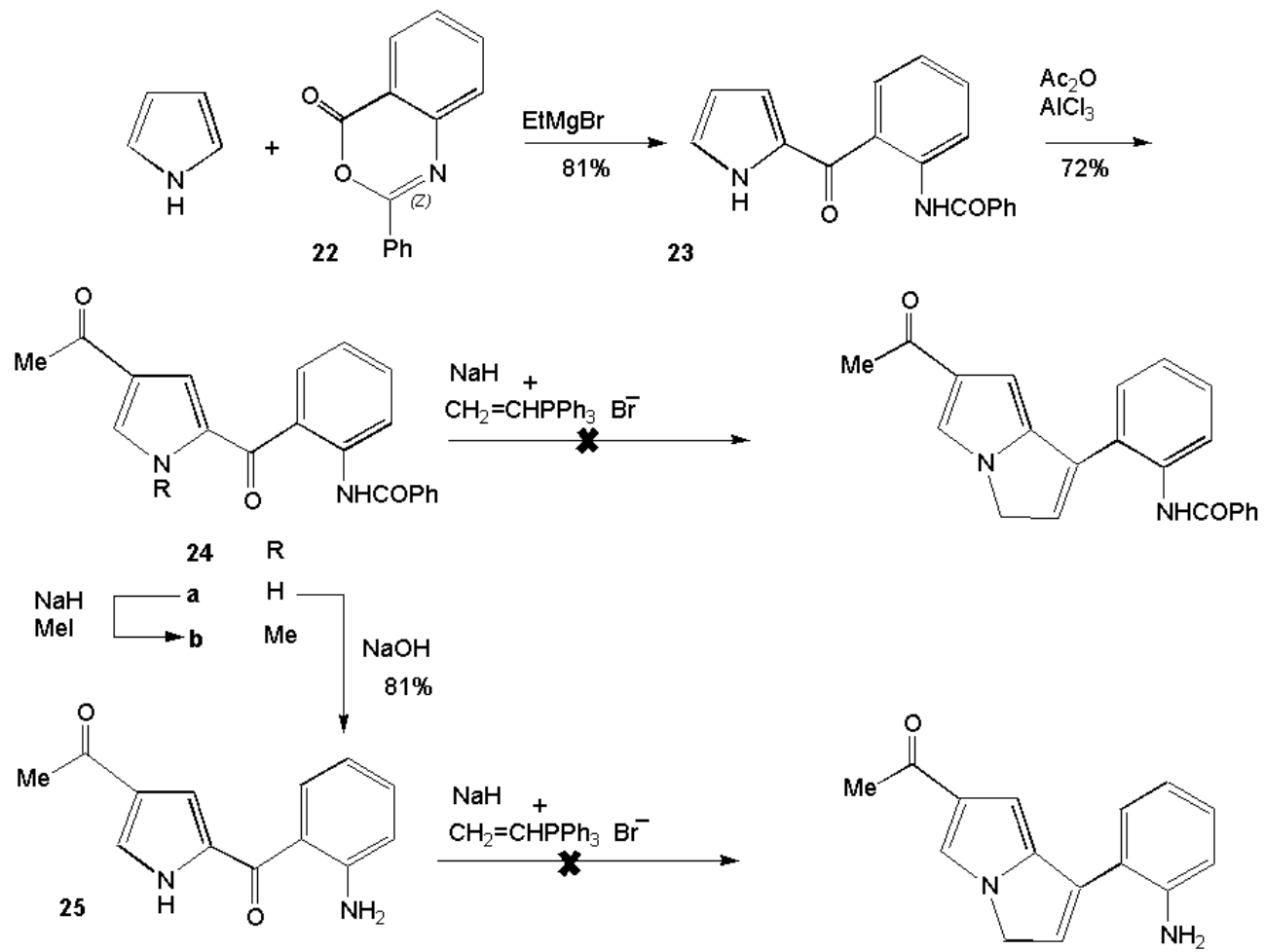

\section{Scheme 7}

It seemed that it would be necessary to mask both of the side-chain $N$-hydrogens. Attempts to convert the amino group in 25 into a 1,3,5-triazine, ${ }^{45,46}$ a phthalimide, or to effect a condensation (Schiff's base) with benzaldehyde, all failed. However, reaction with Bredereck's reagent [t$\mathrm{BuOCH}\left(\mathrm{NMe}_{2}\right)_{2}$ ] converted 25 efficiently into 26. An interesting practical point concerning the relative mobilities on silica gel chromatography of starting primary amine $\mathbf{2 5}$ and product amidine $\mathbf{2 6}$ is that the latter was by far the slower, despite the absence of $N$-hydrogens, presumably because of its greater basicity. To our delight, the Wittig cyclisation strategy was now successful, producing 27 from 26, the relatively low yield undoubtedly reflecting the practical difficulty in separating the product from the triphenylphosphine oxide byproduct. Now, catalytic reduction gave $\mathbf{2 8}$ then conversion of acetyl into ethyl, as before, produced a mixture of 29 and deprotected 30, in unoptimised yields of 12\% and 40\% respectively (Scheme 8). 


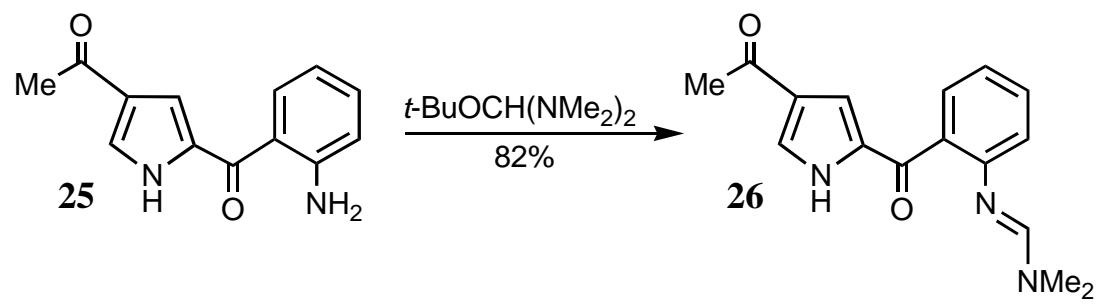<smiles>CNC=Nc1ccccc1C1=CCn2cc(C(=O)O)cc21</smiles><smiles>CCc1cc2n(c1)CCC2c1ccccc1/N=C/N</smiles>

\section{Scheme 8}

\section{Reaction of bicycle 19 with singlet oxygen}

We have so far examined only the ring opening of bicyclic pyrrole $\mathbf{1 9}$ as a model, under the singlet oxygen conditions. In an unoptimised $24 \%$ yield, we obtained the hydroxy-pyrrolidinone 31, as desired. It would now be the plan to convert this into an azadiene 32, perhaps with a suitable silylating agent (Scheme 9).

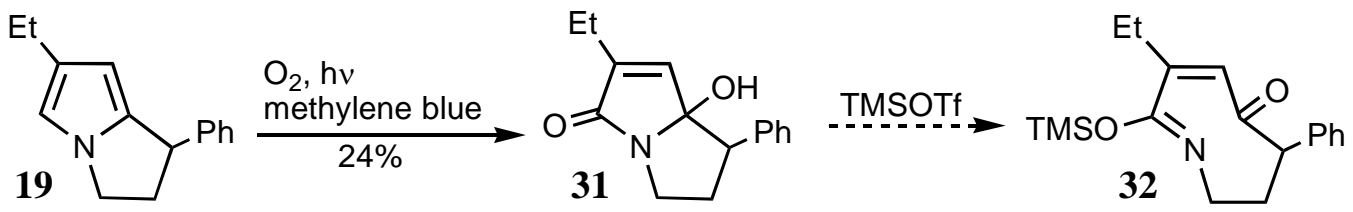

\section{Scheme 9}

\section{Experimental Section}

1-(3-Oxo-3-phenylpropyl)pyrrole (9a). To a stirred solution of 2-aminoethyl phenyl ketone hydrochloride $^{28}(2.5 \mathrm{~g}, 13.5 \mathrm{mmol})$ in water $(22 \mathrm{ml})$ heated at $90{ }^{\circ} \mathrm{C}$, was added sodium ethoxide (1.85 g, $27 \mathrm{mmol}, 2.4$ equiv), acetic acid (12 ml, $0.22 \mathrm{~mol}, 20$ equiv), and 2,5dimethoxytetrahydrofuran $(1.43 \mathrm{ml}, 11.05 \mathrm{mmol}, 1$ equiv). The reaction mixture was heated at 
$100{ }^{\circ} \mathrm{C}$ for $15 \mathrm{~min}$, allowed to cool to rt then extracted with AcOEt (50 ml) and the extract washed with sat. aq. $\mathrm{NaHCO}_{3}\left(3 \times 50 \mathrm{ml}\right.$ ). The organic layer was dried with $\mathrm{MgSO}_{4}$ and evaporated to dryness. Purification by column $\mathrm{SiO}_{2}$ chromatography (30\% of petroleum ether in $\mathrm{CH}_{2} \mathrm{Cl}_{2}$ ) gave 1-(3-oxo-3-phenylpropyl)pyrrole 9a (0.937 g, 42\%) as a brown crystalline solid, mp 74.5-76 ${ }^{\circ} \mathrm{C}$; (found $\mathrm{MH}^{+}$, 200.1073. $\mathrm{C}_{13} \mathrm{H}_{13} \mathrm{NO}$ requires $\mathrm{MH}, 200.1075$ ); $v_{\max }$ (film): 1448, $1681 \mathrm{~cm}^{-1} ;{ }^{1} \mathrm{H}-\mathrm{NMR}\left(300 \mathrm{MHz}, \mathrm{CDCl}_{3}\right): \delta 3.49$ (2H, t, $J$ 7.0, $\left.\mathrm{CH}_{2}\right), 4.42\left(2 \mathrm{H}, \mathrm{t}, J\right.$ 7.0, $\left.\mathrm{CH}_{2}\right), 6.18$ (2H, m, pyrrole $\beta$-Hs), 6.75 (2H, m, pyrrole $\alpha-\mathrm{Hs}), 7.50$ (2H, t, $J$ 8.3, ArH), $7.61(1 \mathrm{H}, \mathrm{m}, \mathrm{ArH})$, 7.96 (2H, d, J 8.3, ArH); ${ }^{13} \mathrm{C}-\mathrm{NMR}\left(75 \mathrm{MHz}, \mathrm{CDCl}_{3}\right): \delta 40.5,44.2,108.3,120.6,127.9,128.6$, 133.4, 136.4, 197.4; m/z (CI): $200\left(\mathrm{MH}^{+}, 100 \%\right)$.

1-(3-Hydroxy-3-phenylpropyl)pyrrole (9b). (a) To a stirred solution of 1-(3-oxo-3phenylpropyl)pyrrole 9a (0.295 g, $1.48 \mathrm{mmol})$ in $i$-PrOH:THF: $\mathrm{H}_{2} \mathrm{O}$ (2:2:1, $\left.25 \mathrm{ml}\right)$ was slowly added $\mathrm{NaBH}_{4}(0.225 \mathrm{~g}, 5.9 \mathrm{mmol}, 4$ equiv). After 45 min when TLC analysis indicated the complete consumption of the starting material, sat. aq. $\mathrm{NH}_{4} \mathrm{Cl}(20 \mathrm{ml})$ was added and the compound extracted with $\mathrm{CH}_{2} \mathrm{Cl}_{2}(3 \times 20 \mathrm{ml})$. The organic layer was dried over $\mathrm{MgSO}_{4}$ and evaporated under reduced pressure. Purification by column $\mathrm{SiO}_{2}$ chromatography $\left(\mathrm{CH}_{2} \mathrm{Cl}_{2}\right)$ provided 1-(3-hydroxy-3-phenylpropyl)pyrrole $\mathbf{9 b}$ (207 mg, 70\%) as a colourless oil; (found $\mathrm{M}^{+}$, 201.1155. $\mathrm{C}_{13} \mathrm{H}_{15} \mathrm{NO}$ requires $\left.M, 201.1154\right) ; v_{\max }$ (film): 701, 727, 1056, 1089, 1455, 1449, 3313-3567 cm ${ }^{-1} ;{ }^{1} \mathrm{H}-\mathrm{NMR}\left(300 \mathrm{MHz}, \mathrm{CDCl}_{3}\right): \delta 2.22\left(2 \mathrm{H}, \mathrm{m}, \mathrm{CH}_{2}\right), 4.08\left(2 \mathrm{H}, \mathrm{m}, \mathrm{CH}_{2}\right), 4.63$ $(1 \mathrm{H}, \mathrm{t}, J$ 4.2, CHOH), 6.20 (2H, d, $J$ 1.3, pyrrole $\beta$-Hs), 6.73 (2H, d, $J 1.3$, pyrrole $\alpha-\mathrm{Hs}), 7.28-$ 7.40 (5H, m, _-ArHs); ${ }^{13} \mathrm{C}-\mathrm{NMR}\left(75 \mathrm{MHz}, \mathrm{CDCl}_{3}\right.$ ): $\delta$ 40.3, 46.1, 71.5, 108.0, 120.5, 125.7, 127.8, 128.6, 143.9; m/z (CI): $202\left(\mathrm{MH}^{+}, 100 \%\right), 184$ (60), 81 (79). (b) To a stirred solution of 3-hydroxy-3-phenylpropanamine ${ }^{31}(3.13 \mathrm{~g}, 20.7 \mathrm{mmol})$ in $\mathrm{AcOH}(300 \mathrm{ml})$, was added NaOAc.3H2O (56.63 g, $414 \mathrm{mmol}, 20$ equiv), and 2,5-dimethoxytetrahydrofuran (2.95 ml, $22.8 \mathrm{mmol}, 1.1$ equiv). The reaction mixture was slowly heated up to $70{ }^{\circ} \mathrm{C}$, kept at that temperature for $2 \mathrm{~h}$ then was concentrated under reduced pressure. $2 \mathrm{M} \mathrm{NaOH}$ was added until a basic $\mathrm{pH}$ was reached and the mixture was extracted with Et2O. The organic layer was dried over $\mathrm{MgSO}_{4}$ and evaporated to dryness. Purification by column $\mathrm{SiO}_{2}$ chromatography (5\% of $\mathrm{Et}_{2} \mathrm{O}$ in petroleum ether) gave 1-(3-hydroxy-3-phenylpropyl)pyrrole $\mathbf{9 b}(1.238 \mathrm{~g}, 30 \%)$ as a colourless oil, with spectroscopic properties identical to those reported above.

1-Phenyl-3-(pyrrol-1-yl)trichloroacetimidate (9c). A solution of 1-(3-hydroxy-3phenylpropyl)pyrrole $\mathbf{9 b}(0.2 \mathrm{~g}, 1 \mathrm{mmol})$ in anhydrous THF $(0.8 \mathrm{ml})$, was added dropwise to a suspension of $\mathrm{NaH}$ (3.5 mg of a $60 \%$ dispersion in mineral oil, $0.087 \mathrm{mmol}$ ) in THF (4 ml). After $15 \mathrm{~min}$, this mixture was added dropwise to a solution of trichloroacetonitrile $(0.1 \mathrm{ml}$, 1 mmol, 1 equiv) in THF ( $1 \mathrm{ml})$ kept between $-5{ }^{\circ} \mathrm{C}$ and $0{ }^{\circ} \mathrm{C}$. The cooling bath was removed after the addition and the solution allowed to warm up to rt. After vigorous stirring, the residue was filtered and evaporated under reduced pressure providing 1-phenyl-3-(pyrrol-1yl)trichloroacetimidate 9c (209 mg, 63\%) as a yellow low mp solid, sufficiently pure for subsequent use; ${ }^{1} \mathrm{H}-\mathrm{NMR}$ (300 MHz, CDCl3): $\delta 2.23\left(1 \mathrm{H}, \mathrm{m}, \mathrm{CH}_{2}\right), 2.41\left(1 \mathrm{H}, \mathrm{m}, \mathrm{CH}_{2}\right), 4.00$ (2H, m, $\mathrm{CH}_{2}$ ), 5.65 (1H, dd, J 9.4, $\left.4.1 \mathrm{~Hz}, \mathrm{CH}\right), 6.10$ (2H, t, $J$ 2.1, pyrrole H), 6.60 (2H, t, $J$ 2.1, pyrrole H), 7.21-7.31 (5H, m, ArH), 8.27 (1H, bs, NH); ${ }^{13} \mathrm{C}-\mathrm{NMR}$ (75 MHz, CDCl3): $\delta 29.6$, 
38.9, 45.6, 108.3, 119.3, 120.4, 125.9, 128.1, 128.5, 139.3, $161.0 \mathrm{~m} / \mathrm{z}$ (CI): $184\left(\mathrm{MH}^{+}-146\right.$ (trichloroacetimidate fragment), 100\%).

2-(Benzotriazol-1-ylmethyl)-4-ethyl-1-(2-(4-methylphenylsulfonyloxy)ethyl)pyrrole (11c). To a stirred solution of 1-propargylbenzotriazole ${ }^{47}(0.706 \mathrm{~g}, 4.5 \mathrm{mmol})$ in THF (22.5 ml) was added a solution of $n$-BuLi (2.81 ml, $1.6 \mathrm{M}, 1$ equiv) at $-78{ }^{\circ} \mathrm{C}$; the solution was stirred at this temperature for $30 \mathrm{~min}$. A solution of 1-bromo-2-butanone $(0.459 \mathrm{ml}, 4.5 \mathrm{mmol}, 1 \mathrm{equiv})$ in THF $(0.4 \mathrm{ml})$ was added, and the reaction mixture was stirred at $-78{ }^{\circ} \mathrm{C}$ for $20 \mathrm{~h}$. Sat. aq. $\mathrm{NH}_{4} \mathrm{Cl}$ (25 ml) was added, and the solution was extracted with $\mathrm{Et}_{2} \mathrm{O}(50 \mathrm{ml})$. The organic phase was separated, washed with sat. aq. $\mathrm{NH}_{4} \mathrm{Cl}\left(3 \times 25 \mathrm{ml}\right.$ ) dried over $\mathrm{MgSO}_{4}$, and concentrated under reduced pressure. The resulting 2-[3-(benzotriazol-1-yl)propyn-1-yl]-2-ethyloxirane was used immediately without characterisation or further purification.

2-[3-(Benzotriazol-1-yl)propyn-1-yl]-2-ethyloxirane $(4.5 \mathrm{mmol})$ was dissolved in $i$-PrOH (30 ml), 2-hydroxyethanamine $(0.539 \mathrm{ml}, 9 \mathrm{mmol}, 2$ equiv) was added, and the solution was refluxed for 24 h. $i-\mathrm{PrOH}$ was removed and the residue subjected to column $\mathrm{SiO}_{2}$ chromatography (25-70\% of AcOEt in petroleum ether) to give 2-(benzotriazol-1-ylmethyl)-4ethyl-1-(2-hydroxyethyl)pyrrole (0.385 g, 32\% from 1-propargylbenzotriazole) as a white foam (found $\mathrm{M}^{+}, 270.1471 . \mathrm{C}_{15} \mathrm{H}_{18} \mathrm{~N}_{4} \mathrm{O}$ requires $M, 270.1481$ ); ${ }^{1} \mathrm{H}-\mathrm{NMR}$ (300 $\mathrm{MHz}, \mathrm{CDCl}_{3}$ ): $\delta 1.10$ (3H, t, $J$ 7.5, $\mathrm{CH}_{3}$ ), 2.52 (2H, q, $J$ 7.5, $\mathrm{CH}_{2}$ ), 3.51 (2H, t, $J$ 5.3, $\mathrm{CH}_{2}$ ), $3.94\left(2 \mathrm{H}, \mathrm{t}, J\right.$ 5.3, $\mathrm{CH}_{2}$ ), $5.76\left(2 \mathrm{H}, \mathrm{s}, \mathrm{CH}_{2}\right), 6.18(1 \mathrm{H}, \mathrm{d}, J 1.9$, pyrrole $\mathrm{H}), 6.42(1 \mathrm{H}, \mathrm{d}, J 1.9$, pyrrole $\mathrm{H}), 7.23-7.42$ (3H, m, ArH), 8.01 (1H, d, J 8.2, ArH); ${ }^{13} \mathrm{C}-\mathrm{NMR}$ (75 $\left.\mathrm{MHz} \mathrm{CDCl}_{3}\right): \delta 15.0,19.8,44.8,48.8,62.5$, 110.2, 110.9, 119.7, 120.3, 123.9, 124.2, 125.7, 127.4, 132.6, 146.0; m/z (CI): $271\left(\mathrm{MH}^{+}, 5 \%\right)$, 152 (95), 120 (54).

To a stirred solution of 2-(benzotriazol-1-ylmethyl)-4-ethyl-1-(2-hydroxyethyl)pyrrole (0.5 g, $1.85 \mathrm{mmol})$ in $\mathrm{CH}_{2} \mathrm{Cl}_{2}(10 \mathrm{ml})$ was added triethylamine (2.77 ml, $19.6 \mathrm{mmol}, 10$ equiv) then $p$-toluenesulfonyl chloride (1.05 g, $5.55 \mathrm{mmol}, 3$ equiv) in portions over a period of $1 \mathrm{~h}$, and the reaction mixture was stirred at room temperature overnight. It was then washed with 2 $\mathrm{M} \mathrm{HCl}(2 \times 10 \mathrm{ml})$, followed by $10 \% \mathrm{NaHCO}_{3}(2 \times 10 \mathrm{ml})$ and water $(3 \times 10 \mathrm{ml})$. The organic layer was dried over $\mathrm{MgSO}_{4}$, and concentrated under reduced pressure. Column $\mathrm{SiO}_{2}$ chromatography gave 2-(benzotriazol-1-ylmethyl)-4-ethyl-1-(2-(4methylphenylsulfonyloxy)ethyl)pyrrole 11c as a colourless oil (0.7 g, 89\%); (found $\mathrm{M}^{+} 424.1563$. $\mathrm{C}_{22} \mathrm{H}_{24} \mathrm{~N}_{4} \mathrm{O}_{3} \mathrm{~S}$ requires $\left.M, 424.1569\right) ;{ }^{1} \mathrm{H}-\mathrm{NMR}\left(300 \mathrm{MHz}, \mathrm{CDCl}_{3}\right.$ ): $\delta 1.10\left(3 \mathrm{H}, \mathrm{t}, J 7.5, \mathrm{CH}_{3}\right.$ ), $2.44\left(5 \mathrm{H}, \mathrm{m}, \mathrm{NCH}_{2}+\mathrm{CH}_{3}\right), 3.96\left(2 \mathrm{H}, \mathrm{t}, J\right.$ 5.2, $\left.\mathrm{CH}_{2}\right), 4.20\left(2 \mathrm{H}, \mathrm{t}, J\right.$ 5.2, $\left.\mathrm{CH}_{2}\right), 5.76\left(2 \mathrm{H}, \mathrm{s}, \mathrm{CH}_{2}\right)$, 6.23 (1H, d, $J$ 1.9, pyrrole H), 6.42 (1H, d, $J$ 1.9, pyrrole H), 7.26 (2H, d, $J$ 8.2, ArH), 7.36 (1H, m, ArH), 7.46 (2H, m, ArH), 7.60 (2H, d, J 8.2, ArH), 8.05 (1H, d, J 8.2, ArH); ${ }^{13} \mathrm{C}-\mathrm{NMR}(75$ $\left.\mathrm{MHz}, \mathrm{CDCl}_{3}\right): \delta 14.9,19.7,21.5,44.4,45.2,69.1,109.9,111.3,119.8,120.1,123.9,124.1$, 126.0, 127.4, 127.7, 129.7, 132.2, 132.5, 144.8, 146.1; m/z (CI): 425 ( $\left.\mathrm{MH}^{+}, 4 \%\right), 306$ (20), 255 (15), 120 (100).

4-Acetyl-2-benzoylpyrrole (16). A solution of benzoyl chloride (0.65 ml, $5.6 \mathrm{mmol}, 1.12$ equiv) in $\mathrm{CH}_{2} \mathrm{Cl}_{2}(2.5 \mathrm{ml})$ was slowly added to a stirred suspension of $\mathrm{AlCl}_{3}(1.60 \mathrm{~g}, 12 \mathrm{mmol}$, 2.4 equiv) in $\mathrm{CH}_{2} \mathrm{Cl}_{2}(10 \mathrm{ml})$ at rt. After $30 \mathrm{~min}$, a solution of 3-acetylpyrrole (0.54 g, $\left.5 \mathrm{mmol}\right)$ was added dropwise during $10 \mathrm{~min}$ and the resulting solution was left stirring for $75 \mathrm{~min}$. The 
mixture was poured into cold water, extracted with $\mathrm{CH}_{2} \mathrm{Cl}_{2}$, the extract washed with aq. $\mathrm{NaHCO}_{3}$, followed by brine, dried over $\mathrm{MgSO}_{4}$, and concentrated under reduced pressure. Column $\mathrm{SiO}_{2}$ chromatography (60\% $\mathrm{Et}_{2} \mathrm{O}$ in petroleum ether) yielded 4-acetyl-2-benzoylpyrrole 16 as a white solid (0.65 g, 60\%), mp 102-103 ${ }^{\circ} \mathrm{C}$, lit ${ }^{41} 103-104{ }^{\circ} \mathrm{C}$; $v_{\max }$ (film): 728, 892, 932, 1181, 1214, 1285, 1376, 1433, 1547, 1626, 1655, $3259 \mathrm{~cm}^{-1} ;{ }^{1} \mathrm{H}-\mathrm{NMR}\left(300 \mathrm{MHz}, \mathrm{CDCl}_{3}\right): \delta$ 2.40 (3H, s, $\left.\mathrm{CH}_{3}\right), 7.23$ (1H, d, J 1.5, pyrrole H), $7.43(2 \mathrm{H}, \mathrm{t}, J$ 7.5, ArH), $7.53(1 \mathrm{H}, \mathrm{m}, \mathrm{ArH})$, 7.67 (1H, d, $J$ 1.5, pyrrole H), 7.8 (2H, dd, $J$ 7.5, 1.5), 10.6 (1H, bs, NH); ${ }^{13} \mathrm{C}-\mathrm{NMR}(75 \mathrm{MHz}$, $\left.\mathrm{CDCl}_{3}\right): \delta 27.3,118.2,127.7,128.4,128.5,128.9,131.7,132.5,137.2,185.4,193.2 ; \mathrm{m} / \mathrm{z}(\mathrm{CI})$ : $231\left(\mathrm{MNH}_{4}^{+}, 20 \%\right), 213\left(\mathrm{MH}^{+}, 100\right)$.

6-Acetyl-1-phenyl-3H-pyrrolizine (17). To a stirred suspension of 4-acetyl-2-benzoylpyrrole 16 (2.13 g, $10 \mathrm{mmol}$ ), and vinyltriphenylphosphonium bromide (3.69 g, $10 \mathrm{mmol}$, 1 equiv) in anhydrous THF $(200 \mathrm{ml})$ at $-10^{\circ} \mathrm{C}, \mathrm{NaH}(0.56 \mathrm{~g}$ of a $60 \%$ dispersion in mineral oil, $14 \mathrm{mmol}$, 1.4 equiv) was added. After $15 \mathrm{~min}$, the reaction mixture was poured into cold water, extracted with $\mathrm{Et}_{2} \mathrm{O}$, the extract washed with brine, dried over $\mathrm{MgSO}_{4}$, and concentrated under reduced pressure. Column $\mathrm{SiO}_{2}$ chromatography (25\% of AcOEt in hexane) yielded 6-acetyl-1-phenyl$3 \mathrm{H}$-pyrrolizine 17 as a clear yellow oil (1.4 g, 65\%); (found $\mathrm{M}^{+}, 223.0996 . \mathrm{C}_{15} \mathrm{H}_{13} \mathrm{NO}$ requires $M, 223.0997)$; $v_{\max }$ (film): 741, 1072, 1185, 1198, 1258, 1375, 1445, 1491, 1506, $1640 \mathrm{~cm}^{-1}$; ${ }^{1} \mathrm{H}-$ NMR (300 MHz, $\left.\mathrm{CDCl}_{3}\right): \delta 2.48\left(3 \mathrm{H}, \mathrm{s}, \mathrm{CH}_{3}\right), 4.68\left(2 \mathrm{H}, \mathrm{d}, J\right.$ 2.3, $\left.\mathrm{CH}_{2}\right), 6.50(1 \mathrm{H}, \mathrm{t}, J$ 2.3, CH), 6.70 (1H, s, pyrrole H), 7.46 (3H, m, ArH), $7.64(1 \mathrm{H}$, s, pyrrole $\mathrm{H}), 7.75(2 \mathrm{H}, \mathrm{d}, J 7.5, \mathrm{ArH}) ;{ }^{1} \mathrm{H}-$ NMR (300 MHz, $\left.\left(\mathrm{CD}_{3}\right)_{2} \mathrm{CO}\right): \delta 2.34\left(3 \mathrm{H}, \mathrm{s}, \mathrm{CH}_{3}\right), 4.74\left(2 \mathrm{H}, \mathrm{bs}, \mathrm{CH}_{2}\right), 6.63(1 \mathrm{H}, \mathrm{s}$, pyrrole $\mathrm{H})$, 6.73 (1H, t, J 2.3, CH), 7.39 (1H, m, ArH), 7.47 (2H, t, J 7.3, ArH), 7.78 (1H, s, pyrrole H), 7.80 (2H, d, J 7.3, ArH); ${ }^{13} \mathrm{C}-\mathrm{NMR}\left(75 \mathrm{MHz},\left(\mathrm{CD}_{3}\right)_{2} \mathrm{CO}\right): \delta$ 26.4, 52.1, 98.3, 123.2, 124.6, 126.5, 128.7, 129.0, 130.2, 133.0,138.0, 140.9, 192.0; m/z (CI): $241\left(\mathrm{MNH}_{4}^{+}, 1 \%\right), 224\left(\mathrm{MH}^{+}, 100\right)$, 214 (4), 182 (1).

6-Acetyl-2,3-dihydro-1-phenyl-1H-pyrrolizine (18). To a stirred solution of 1-phenyl-6acetyl-pyrrolizine 17 (2.67 g, $11.9 \mathrm{mmol})$ in $\mathrm{MeOH}$ (30 ml) was added $\mathrm{Pd} / \mathrm{C}(0.27 \mathrm{~g}, 10 \%)$. The resulting mixture was purged three times with $\mathrm{H}_{2}$, and left under a $\mathrm{H}_{2}$ atmosphere overnight. The mixture was then purged with $\mathrm{N}_{2}$, passed through a pad of celite, and evaporated to dryness. Column $\mathrm{SiO}_{2}$ chromatography (30\% of EtOAc in hexane) yielded 6-acetyl-2,3-dihydro-1phenyl-1H-pyrrolizine 18 as a clear oil (2.67 g, 98\%); (found $\mathrm{M}^{+}$, 225.1157. $\mathrm{C}_{15} \mathrm{H}_{15} \mathrm{NO}$ requires M, 225.1154); $v_{\max }$ (film): 751, 936, 1064, 1207, 1286, 1371, 1426, 1447, 1469, 1509, 1547, $1650 \mathrm{~cm}^{-1}$; ${ }^{1} \mathrm{H}-\mathrm{NMR}\left(300 \mathrm{MHz}, \mathrm{CDCl}_{3}\right): \delta 2.49\left(3 \mathrm{H}, \mathrm{s}, \mathrm{CH}_{3}\right), 2.51(1 \mathrm{H}, \mathrm{m}, \mathrm{CHH}), 2.99(1 \mathrm{H}, \mathrm{m}$, $\mathrm{CHH}), 4.05$ (1H, m, CHH), 4.16 (1H, m, CHH), 4.40 (1H, t, J 7.7, CH), 6.29 (1H, s, H pyrrole), 7.25-7.31 (3H, m, ArH), 7.29 (1H, s, H pyrrole), 7.33-36 (2H, d, J 7.5, ArH); ${ }^{1} \mathrm{H}-\mathrm{NMR}(300$ $\left.\mathrm{MHz},\left(\mathrm{CD}_{3}\right)_{2} \mathrm{CO}\right): \delta 2.35\left(3 \mathrm{H}, \mathrm{s}, \mathrm{CH}_{3}\right), 2.40(1 \mathrm{H}, \mathrm{m}, \mathrm{CHH}), 2.92(1 \mathrm{H}, \mathrm{m}, \mathrm{CHH}), 4.02(1 \mathrm{H}, \mathrm{m}$, $\mathrm{CHH}), 4.15$ (1H, m, CHH), 4.32 (1H, t, J 7.7, CH), 6.12 (1H, s, H pyrrole), 7.20 (3H, t, ArH), 7.27 ( $2 \mathrm{H}, \mathrm{m}, \mathrm{ArH}), 7.52$ (1H, s, H pyrrole); ${ }^{13} \mathrm{C}-\mathrm{NMR}$ (75 MHz, $\left.\left(\mathrm{CD}_{3}\right)_{2} \mathrm{CO}\right): \delta 26.8,38.5,43.0$, 45.8, 101.2, 119.4, 126.8, 127.2, 128.6, 130.3, 140.8, 142.2, 193.7; m/z (CI): $226\left(\mathrm{M}+\mathrm{H}^{+}\right.$, 100\%), 214 (5).

6-Ethyl-2,3-dihydro-1-phenyl-1H-pyrrolizine (19). To a stirred suspension of $\mathrm{AlCl}_{3}(0.23 \mathrm{~g}$, 1.73, 3 equiv) in $\mathrm{CH}_{2} \mathrm{Cl}_{2}(7 \mathrm{ml})$ at rt was added $t-\mathrm{BuNH}_{2} \mathrm{BH}_{3}(0.23 \mathrm{~g}, 1.73 \mathrm{mmol}, 3$ equiv). The 
mixture was stirred for $30 \mathrm{~min}$ and a solution of 6-acetyl-2,3-dihydro-6-phenyl- $1 \mathrm{H}$-pyrrolizine 18 (0.13 g, $0.57 \mathrm{mmol})$ in $\mathrm{CH}_{2} \mathrm{Cl}_{2}(3 \mathrm{ml})$ was added. After $2 \mathrm{~h}$ the reaction mixture was poured onto ice and extracted with $\mathrm{CH}_{2} \mathrm{Cl}_{2}$. The combined organic extracts were rapidly washed with dil. citric acid, sat. aq. $\mathrm{NaHCO}_{3}$, then brine, dried over $\mathrm{MgSO}_{4}$ and concentrated under reduced pressure. Column $\mathrm{SiO}_{2}$ chromatography (10\% of EtOAc in hexane) yielded 6-ethyl-2,3-dihydro1-phenyl-1H-pyrrolizine 19 as a clear oil (0.9 g, 74\%); (found $\mathrm{M}^{+}, 211.1357 \mathrm{C}_{15} \mathrm{H}_{17} \mathrm{~N}$ requires $M, 211.1361)$; ${ }^{1} \mathrm{H}-\mathrm{NMR}$ (300 MHz, $\mathrm{CDCl}_{3}$ ): $\delta 1.12$ (3H, t, J 7.5, $\mathrm{CH}_{3}$ ), $2.28(1 \mathrm{H}, \mathrm{m}, \mathrm{CHH}), 2.44$ (2H, q, J 7.5, CH $\mathrm{CH}_{2}, 2.81(1 \mathrm{H}, \mathrm{m}, \mathrm{CHH}), 3.84(1 \mathrm{H}, \mathrm{m}, \mathrm{CHH}), 3.95(1 \mathrm{H}, \mathrm{m}, \mathrm{CHH}), 4.25(1 \mathrm{H}, \mathrm{t}, J$ 7.7, CH), $5.20(1 \mathrm{H}$, s, pyrrole $\mathrm{H}), 6.40(1 \mathrm{H}$, s, pyrrole $\mathrm{H}), 7.14-7.25(5 \mathrm{H}, \mathrm{m}, \mathrm{ArH}) ;{ }^{1} \mathrm{H}-\mathrm{NMR}$ (300 MHz, $\left.\left(\mathrm{CD}_{3}\right)_{2} \mathrm{CO}\right): \delta 1.05$ (3H, t, $J$ 7.5, $\left.\mathrm{CH}_{3}\right), 2.20(1 \mathrm{H}, \mathrm{m}, \mathrm{CHH}), 2.35$ (2H, q, $J$ 7.5, $\mathrm{CH}_{2}$ ), $2.80(1 \mathrm{H}, \mathrm{m}, \mathrm{CHH}), 3.80(1 \mathrm{H}, \mathrm{m}, \mathrm{CHH}), 3.95(1 \mathrm{H}, \mathrm{m}, \mathrm{CHH}), 4.20(1 \mathrm{H}, \mathrm{t}, J$ 7.7, CH), $5.43(1 \mathrm{H}$, s, H pyrrole), 6.35 (1H, s, H pyrrole), 7.15 (5H, m, ArH); m/z (CI): 212 ( $\left.\mathrm{MH}^{+}, 100 \%\right), 186$ (62), 96 (20).

2-(2-Nitrobenzoyl)-1-phenylsulfonylpyrrole (20). To a stirred solution of $\mathrm{SnCl}_{4}(9.4 \mathrm{ml}$, $36.22 \mathrm{mmol}, 1.5$ equiv) in anhydrous 1,2-dichloroethane $(150 \mathrm{ml})$ at $-10{ }^{\circ} \mathrm{C}$, was added slowly o-nitrobenzoyl chloride $(6.27 \mathrm{~g}, 33.81 \mathrm{mmol}, 1.4$ equiv). The resulting mixture was stirred for $10 \mathrm{~min}$. A solution of 1-phenylsulfonylpyrrole ${ }^{48}$ (5 g, $\left.24.2 \mathrm{mmol}\right)$ in 1,2-dichloroethane (100 ml) was added dropwise, and the mixture stirred at $-10{ }^{\circ} \mathrm{C}$ for $30 \mathrm{~min}$ and at rt for $90 \mathrm{~min}$. The reaction was then poured into ice water and extracted with $\mathrm{CH}_{2} \mathrm{Cl}_{2}$. The combined organic extracts were washed with sat. aq. $\mathrm{NaHCO}_{3}$, then brine, dried over $\mathrm{MgSO}_{4}$ and concentrated under reduced pressure. Purification by column $\mathrm{SiO}_{2}$ chromatography (10-20-30\% of AcOEt in hexane) afforded 2-(2-nitrobenzoyl)-1-phenylsulfonylpyrrole 20 as a white solid (4.87 g, 57\%), mp 133-134 ${ }^{\circ} \mathrm{C}$; (found $\mathrm{MH}^{+}$, 357.0549. $\mathrm{C}_{17} \mathrm{H}_{12} \mathrm{~N}_{2} \mathrm{SO}_{5}$ requires $M H, 357.0554$ ); $v_{\max }$ (film): 726, 1061, 1154, 1346, 1448, 1429, 1527, 1663, $3649 \mathrm{~cm}^{-1} ;{ }^{1} \mathrm{H}-\mathrm{NMR}$ (300 MHz, $\mathrm{CDCl}_{3}$ ): $\delta 6.34$ (1H, m, pyrrole H), 6.50 (1H, dd, $J$ 3.8, 1.8 pyrrole $\mathrm{H}), 7.51(2 \mathrm{H}$, dd, $J$ 7.7, $1.6 \mathrm{ArH}), 7.67$ (4H, m, ArH), 7.97 (1H, dd, $J$ 3.2, 1.8, pyrrole H), 8.12 (3H, m, ArH); ${ }^{13} \mathrm{C}-\mathrm{NMR}\left(75 \mathrm{MHz}, \mathrm{CDCl}_{3}\right): \delta$ 110.7, 122.0, 124.3, 126.8, 128.4, 128.8, 129.2, 130.6, 131.4, 133.6, 134.0, 135.3, 146.7, 179.8; $\mathrm{m} / \mathrm{z}$ (CI): $374\left(\mathrm{MNH}_{4}{ }^{+}, 42 \%\right), 357\left(\mathrm{MH}^{+}, 100\right), 93$ (50).

2-(2-Nitrobenzoyl)pyrrole. To a stirred solution of 1-(phenylsulfonyl)-2-(2nitrobenzoyl)pyrrole 20 (2.7 g, $7.5 \mathrm{mmol})$ in $\mathrm{MeOH}(150 \mathrm{ml})$ was added $2 \mathrm{M} \mathrm{NaOH}(6 \mathrm{ml}$, $12 \mathrm{mmol}, 1.6)$ equiv and the resulting mixture was refluxed for $4 \mathrm{~h}$. After cooling to rt, the solvent was evaporated under reduced pressure, water was added (15 ml) and the $\mathrm{pH}$ adjusted to 4-5 by dropwise addition of $2 \mathrm{M} \mathrm{HCl}$. The suspension was extracted with $\mathrm{CHCl}_{3}$ and the combined organic extracts washed with brine, dried over $\mathrm{MgSO}_{4}$ and concentrated to give 2-(2nitrobenzoyl)pyrrole as a yellowish solid (1.23 g, 74\%). An analytical sample was purified by column $\mathrm{SiO}_{2}$ chromatography (20\% of AcOEt in hexane), mp 137-139 ${ }^{\circ} \mathrm{C}$, lit $43138-140{ }^{\circ} \mathrm{C}$; $v_{\max }$ (film): 895, 1347, 1401, 1526, 1625, $3292 \mathrm{~cm}^{-1}$; ${ }^{1} \mathrm{H}-\mathrm{NMR}$ (300 MHz, $\left.\mathrm{CDCl}_{3}\right): \delta 6.31$ (1H, m, pyrrole H), $6.51(1 \mathrm{H}, \mathrm{m}$, pyrrole $\mathrm{H}), 7.21(1 \mathrm{H}, \mathrm{m}$, pyrrole $\mathrm{H}), 7.63(1 \mathrm{H}, \mathrm{td}, J$ 7.4, $1.6 \mathrm{ArH}), 7.70$ (1H, dd, $J$ 8.0, 1.6, ArH), 7.76 (1H, td, $J$ 7.4, 1.4, ArH), 8.18 (1H, dd, $J$ 8.0, 1.4, ArH), 9.75 (1H, 
bs, NH); ${ }^{13} \mathrm{C}-\mathrm{NMR}\left(75 \mathrm{MHz}, \mathrm{CD}_{3} \mathrm{CO}\right): \delta$ 110.8, 119.2, 124.7, 127.0, 129.7, 131.1, 131.2, 133.8, 135.6, 148.1, 181.2; m/z (CI): $234\left(\mathrm{MNH}_{4}{ }^{+}, 100 \%\right), 217\left(\mathrm{MH}^{+}, 10\right), 187$ (24).

4-Acetyl-2-(2-nitrobenzoyl)pyrrole (21). To a stirred suspension of $\mathrm{AlCl}_{3}(2.46 \mathrm{~g}, 18.5 \mathrm{mmol}$, 4 equiv) in 1,2-dichloroethane ( $50 \mathrm{ml})$, acetic anhydride ( $1.3 \mathrm{ml}, 13.8 \mathrm{mmol}, 3$ equiv) was added slowly. After $30 \mathrm{~min}$ stirring at rt, a solution of 2-(2-nitrobenzoyl)pyrrole (1 g, $4.6 \mathrm{mmol}$ ) in 1,2dichloroethane $(10 \mathrm{ml})$ was added and the reaction mixture was left at reflux overnight. The reaction mixture was poured onto ice water, extracted with $\mathrm{CH}_{2} \mathrm{Cl}_{2}$, the extract washed with brine, dried over $\mathrm{MgSO}_{4}$ and concentrated. Column $\mathrm{SiO}_{2}$ chromatography (25-50\% of AcOEt in hexane) afforded 4-acetyl-2-(2-nitrobenzoyl)pyrrole 21 as an orange solid (0.81 g, 68\%), $\mathrm{mp}$ $151{ }^{\circ} \mathrm{C}$; (found $\mathrm{M}^{+}$, 258.0637. $\mathrm{C}_{13} \mathrm{H}_{10} \mathrm{~N}_{2} \mathrm{O}_{4}$ requires $M$, 258.0641); $v_{\max }$ (film): 708, 1347, 1377, 1526, 1551, $1637 \mathrm{~cm}^{-1}$; ${ }^{1} \mathrm{H}-\mathrm{NMR}\left(300 \mathrm{MHz}, \mathrm{CDCl}_{3}\right): \delta 2.44\left(3 \mathrm{H}, \mathrm{s}, \mathrm{CH}_{3}\right), 6.87(1 \mathrm{H}, \mathrm{s}$, pyrrole H), $7.60(1 \mathrm{H}, \mathrm{d}, J$ 7.3, ArH), $7.74(1 \mathrm{H}, \mathrm{s}$, pyrrole H), $7.76(2 \mathrm{H}, \mathrm{m}, \mathrm{ArH}), 8.18(1 \mathrm{H}, \mathrm{d}, J$ 8.0, ArH), $9.84(1 \mathrm{H}, \mathrm{bs}, \mathrm{NH}) ;{ }^{1} \mathrm{H}-\mathrm{NMR}\left(300 \mathrm{MHz},\left(\mathrm{CD}_{3}\right)_{2} \mathrm{CO}\right): \delta 2.20\left(3 \mathrm{H}, \mathrm{s}, \mathrm{CH}_{3}\right), 6.90(1 \mathrm{H}, \mathrm{s}$, pyrrole H), 7.75 (1H, d, J 7.3, ArH), 7.92 (3H, m, pyrrole $\mathrm{H}+\mathrm{ArH}), 8.25$ ( $1 \mathrm{H}, \mathrm{d}, J$ 8.0, ArH); ${ }^{13} \mathrm{C}-\mathrm{NMR}$ (75 MHz, $\left.\mathrm{CD}_{3} \mathrm{CO}\right): \delta$ 26.7, 117.5, 124.9, 128.1, 129.6, 130.0, 131.9, 133.1, 134.2, 135.6, 139.4, 182.5, 192.2; m/z (CI): $276\left(\mathrm{MNH}_{4}{ }^{+}, 46 \%\right), 259\left(\mathrm{MH}^{+}\right.$, 5), 229 (100).

2-Phenyl-4H-benzoxazin-4-one (22). To a stirred mixture of anthranilic acid (8.88 g, $64.8 \mathrm{mmol})$ in THF $(130 \mathrm{ml})$ at $0-5{ }^{\circ} \mathrm{C}, \mathrm{Na}_{2} \mathrm{CO}_{3}$ (powder, $13.73 \mathrm{~g}, 129.6 \mathrm{mmol}, 2$ equiv) was added followed by benzoyl chloride ( $18.8 \mathrm{ml}, 162 \mathrm{mmol}, 2.5$ equiv). After $10 \mathrm{~min}$, the cold bath was removed and the mixture was stired at rt overnight. Water $(130 \mathrm{ml})$, was added and the mixture was stirred for 10 min prior to filtration. The solid was washed with water and then $50 \%$ aq. $\mathrm{MeOH}$. The additional material precipitated from the filtrate was collected by filtration and washed. The combined crops were dried at $50{ }^{\circ} \mathrm{C}$ under high vacuum to give 2-phenyl- $4 \mathrm{H}$ benzoxazin-4-one 22 (14.2 g, 98\%) as a white solid, mp $119{ }^{\circ} \mathrm{C}$, lit ${ }^{49} 124{ }^{\circ} \mathrm{C} ; \Lambda_{\max }$ (film): 765, 1257, 1688, $1762 \mathrm{~cm}^{-1}$; ${ }^{1} \mathrm{H}-\mathrm{NMR}$ (300 MHz, $\left.\mathrm{CDCl}_{3}\right)$ : $\not \subset 7.55$ (4H, m, ArH), 7.72 (1H, dd, J 8.2, 0.69, ArH), 7.86 (1H, dt, $J$ 7.6, 1.6, ArH), 8.27 (1H, dd, $J$ 7.6, 1.5, ArH), 8.34 (2H, dd, $J$ 8.2, 1.5, ArH); ${ }^{13} \mathrm{C}-\mathrm{NMR}\left(75 \mathrm{MHz}, \mathrm{CDCl}_{3}\right): \not \subset 127.1,128.1,128.2,128.7,130.1,132.5,133.7$, 136.5, 146.9, 157.4, 159.8, 171.2; m/z (CI): 224 ( $\left.\mathrm{MH}^{+}, 100 \%\right), 105$ (20).

$\mathrm{N}$-[2-(1H-Pyrrol-2-ylcarbonyl)phenyl]benzamide (23). After a solution of EtMgCl (52.5 ml, 2 M solution in THF, $105 \mathrm{mmol}, 2.1$ equiv) in anhydrous THF $(20 \mathrm{ml})$ was cooled to $0{ }^{\circ} \mathrm{C}$, a solution of freshly distilled pyrrole $(7.98 \mathrm{ml}, 115 \mathrm{mmol}, 2.3$ equiv) in dry toluene $(7.98 \mathrm{ml})$ was added dropwise over $20 \mathrm{~min}$, keeping the mixture in an ice bath. After the mixture was stirred to rt for $20 \mathrm{~min}$, a suspension of 2-phenyl-4H-benzoxazin-4-one 22 (11.15 g, $50 \mathrm{mmol})$ in THF (35 ml) was added. After 45 min stirring, the mixture was refluxed for 3 h. Sat. aq. $\mathrm{NH}_{4} \mathrm{Cl}$ (11.5 ml) was then added to the hot mixture over 5 min. After 20 min of stirring, $\mathrm{Na}_{2} \mathrm{SO}_{4}$ (11.5 g) was added. The suspension was stirred for $20 \mathrm{~min}$ prior to filtration. The collected solid was washed with THF, and the combined organic filtrate and washes were concentrated to dryness. The residue was suspended in toluene $(50 \mathrm{ml})$, and the suspension cooled in an ice bath for $20 \mathrm{~min}$. The solid was collected by filtration and washed with hexane. Drying at rt overnight under high vacuum gave $N$-[2-(1H-pyrrol-2-ylcarbonyl)phenyl]benzamide 23 as light green crystals $(11.7 \mathrm{~g}, 81 \%)$. An analytical sample was recrystallised from acetone, mp $185{ }^{\circ} \mathrm{C}$, lit ${ }^{50}$ 
(mp not given); $v_{\max }$ (film): 760, 888, 1127, 1500, 1335, 1393, 1421, 1513, 1590, 1658, $3277 \mathrm{~cm}^{-1}$; ${ }^{1} \mathrm{H}-\mathrm{NMR}\left(300 \mathrm{MHz}, \mathrm{CDCl}_{3}\right): \delta 6.28(1 \mathrm{H}, \mathrm{m}$, pyrrole $\mathrm{H}), 6.81(1 \mathrm{H}, \mathrm{m}$, pyrrole $\mathrm{H})$, $7.17(1 \mathrm{H}, \mathrm{m}$, pyrrole H), 7.23 (1H, dt, 7.8, 0.8, ArH), $7.42(3 \mathrm{H}, \mathrm{m}, \mathrm{ArH}), 7.53(1 \mathrm{H}, \mathrm{t}, J$ 7.8, ArH), 7.91 (3H, dt, $J$ 7.8, 1.5, ArH), 8.69 (1H, d, J 8.3, 0.8, ArH), 9.63 (1H, bs, NH), 11.04 (1H, bs, $\mathrm{NH}) ;{ }^{13} \mathrm{C}-\mathrm{NMR}\left(75 \mathrm{MHz}, \mathrm{CDCl}_{3}\right): \delta 111.5,120.9,121.5,122.5,124.5,126.1,127.2,128.7$, 131.3, 131.4, 131.8, 133.2, 134.7, 139.5, 165.5, 186.1; m/z (CI): $308\left(\mathrm{MNH}_{4}^{+}, 13 \%\right), 291\left(\mathrm{MH}^{+}\right.$, 100), 124 (46), 105 (92), 68 (79).

$\mathrm{N}$-[2-(4-Acetyl-1H-pyrrol-2-ylcarbonyl)phenyl]benzamide (24a). Acetic anhydride (6.1 ml, $64.68 \mathrm{~g}, 2.68$ equiv) was added at $0{ }^{\circ} \mathrm{C}$ to a suspension of aluminium chloride (19.31 g, $144.8 \mathrm{mmol}, 6$ equiv) in $\mathrm{CH}_{2} \mathrm{Cl}_{2}(50 \mathrm{ml})$. After $30 \mathrm{~min}$ of stirring at $\mathrm{rt}$, the reaction mixture was cooled at $0{ }^{\circ} \mathrm{C}$ and then a solution of $\mathrm{N}$-[2-(1H-pyrrol-2-ylcarbonyl)phenyl]benzamide 23 (7 g, $24.13 \mathrm{mmol}$ ) in $\mathrm{CH}_{2} \mathrm{Cl}_{2}$ (50 ml) was added and the reaction was allowed to warm to rt overnight. The reaction mixture was poured into cold water, extracted with $\mathrm{CH}_{2} \mathrm{Cl}_{2}$, the extract washed with brine, dried over $\mathrm{MgSO}_{4}$ and concentrated under reduced pressure. Column $\mathrm{SiO}_{2}$ chromatography $(50-100 \%$ of EtOAc in hexane) gave $\mathrm{N}$-[2-(4-acetyl-1H-pyrrol-2ylcarbonyl)phenyl]benzamide 24a (5.75 g, 72\%) as a white crystalline solid, mp $179{ }^{\circ} \mathrm{C}$; (found $\mathrm{M}^{+}$, 332.1161. $\mathrm{C}_{20} \mathrm{H}_{16} \mathrm{~N}_{2} \mathrm{O}_{3}$ requires $M$, 332.1161); $v_{\max }$ (film): 755, 1076, 1247, 1298, 1407, 1445, 1524, 1590, 1678, 3649, $3854 \mathrm{~cm}^{-1} ;{ }^{1} \mathrm{H}-\mathrm{NMR}$ (300 MHz, $\left.\mathrm{CDCl}_{3}\right): \delta 2.38\left(3 \mathrm{H}, \mathrm{s}, \mathrm{CH}_{3}\right)$, 7.15 (1H, t, $J$ 7.8, ArH), 7.18 (1H, m, pyrrole H), 7.43 (3H, m, ArH), 7.56 (1H, t, $J$ 7.8, ArH), 7.61 (1H, m, pyrrole H), 7.92 (3H, d, $J$ 7.8, ArH), 8.69 (1H, d, $J$ 8.3, ArH), 10.06 (1H, bs, NH), 11.37 (1H, bs, $\mathrm{NH}) ;{ }^{13} \mathrm{C}-\mathrm{NMR}\left(75 \mathrm{MHz}, \mathrm{CDCl}_{3}\right): \delta 27.3,119.0,121.6,122.8,123.4,127.2$, 127.9, 128.3, 128.5, 128.7, 131.4, 131.9, 134.1, 134.5, 139.8, 165.5, 186.6, 193.1; m/z (EI): 332 (M+17\%), 196 (33), 105 (21), 49 (100).

$\mathrm{N}$-[2-(4-Acetyl-1-methylpyrrol-2-ylcarbonyl)phenyl]benzamide (24b). To a stirred solution of $\mathrm{N}$-[2-(4-acetyl-1H-pyrrol-2-ylcarbonyl)phenyl]benzamide $24 \mathrm{a}(0.15 \mathrm{~g}, 0.45 \mathrm{mmol})$ in anhydrous THF $(10 \mathrm{ml})$ at $0{ }^{\circ} \mathrm{C}$, $\mathrm{NaH}(0.019 \mathrm{~g}, 60 \%$ dispersion in mineral oil, $0.495 \mathrm{mmol}, 1.1$ equiv) was added and the reaction was left stirring for $30 \mathrm{~min}$ before the addition of methyl iodide $(0.028 \mathrm{ml}, 0.45 \mathrm{mmol}, 1$ equiv). The reaction mixture was allowed to warm up to $\mathrm{rt}$ overnight. It was then poured into cold water, product extracted into $\mathrm{CH}_{2} \mathrm{Cl}_{2}$, the extract washed with brine, dried over $\mathrm{MgSO}_{4}$ and concentrated under reduced pressure. Column $\mathrm{SiO}_{2}$ chromatography (25-50\% of EtOAc in hexane) afforded $\mathrm{N}$-[2-(4-acetyl-1-methylpyrrol-2ylcarbonyl)phenyl]benzamide 24b (0.075 g, 50\%) as a white solid, mp $140{ }^{\circ} \mathrm{C}$; (found $\mathrm{M}^{+}$, 346.1312. $\mathrm{C}_{21} \mathrm{H}_{18} \mathrm{~N}_{2} \mathrm{O}_{3}$ requires $M$, 346.1317); $v_{\max }$ (film): 759, 919, 1196, 1268, 1384, 1447, 1525, 1580, 1597, $1670 \mathrm{~cm}^{-1}$; ${ }^{1} \mathrm{H}-\mathrm{NMR}\left(300 \mathrm{MHz}, \mathrm{CDCl}_{3}\right): \delta 2.45\left(3 \mathrm{H}, \mathrm{s}, \mathrm{CH}_{3}\right), 4.09(3 \mathrm{H}, \mathrm{s}$, $\left.\mathrm{NCH}_{3}\right), 7.09(1 \mathrm{H}, \mathrm{d}, J$ 1.9, pyrrole H), $7.22(1 \mathrm{H}, \mathrm{dt}, J$ 7.5, 1.1, ArH), $7.56(4 \mathrm{H}, \mathrm{m}, 3 \mathrm{ArH}$ and pyrrole H), 7.67 (1H, t, $J$ 7.8, ArH), 7.87 (3H, dd, $J$ 7.8, 1.5, ArH), 8.04 (2H, dd, J 8.0, 1.5), 8. 80 (1H, d, $J$ 8.0, $1.5 \mathrm{ArH}), 11.40$ (1H, bs, NH); ${ }^{13} \mathrm{C}-\mathrm{NMR}\left(75 \mathrm{MHz}, \mathrm{CDCl}_{3}\right): \delta 27.1,37.9,121.6$, 122.4, 122.5, 124.6, 124.9, 127.2, 128.7, 131.3, 131.9, 132.2, 133.7, 134.1, 134.6, 139.8, 165.5, 184.6, 192.7; m/z (CI): $347\left(\mathrm{MH}^{+}, 100 \%\right), 223$ (40), 124 (79), 104 (78), 94 (64), 74 (72), 58 (99). 
2-Aminophenyl 4-acetylpyrrol-2-yl ketone (25). A mixture of $\mathrm{N}$-[2-(4-acetyl-1H-pyrrol-2ylcarbonyl)phenyl]benzamide 24a (2.18 g, $6.3 \mathrm{mmol}, 1$ equiv), $10 \mathrm{M} \mathrm{NaOH}$ (2 ml) and $\mathrm{MeOH}$ $(7 \mathrm{ml})$ was heated at reflux overnight. Water $(10 \mathrm{ml})$ was added and the mixture was slowly cooled down to rt and left stirring for $3 \mathrm{~h}$ ( $\mathrm{pH}$ was adjusted to neutral). The reaction mixture was extracted with $\mathrm{CH}_{2} \mathrm{Cl}_{2}$, the extract washed with brine, dried over $\mathrm{MgSO}_{4}$ and concentrated under reduced pressure. Column $\mathrm{SiO}_{2}$ chromatography (25\% of EtOAc in hexane) gave 2-aminophenyl 4-acetylpyrrol-2-yl ketone 25 (1.21 g, 81\%) as a yellow solid, mp 129-130 ${ }^{\circ} \mathrm{C}$; (found $\mathrm{M}^{+}$, 228.0898. $\mathrm{C}_{13} \mathrm{H}_{12} \mathrm{~N}_{2} \mathrm{O}_{2}$ requires $\left.M, 228.0899\right) ; v_{\max }$ (film): 1212, 1259, 1293, 1379, 1430, 1546, 1582, 1614, 1655, 3261, $3347 \mathrm{~cm}^{-1}$; ${ }^{1} \mathrm{H}-\mathrm{NMR}$ (300 MHz, $\left.\mathrm{CDCl}_{3}\right): \delta 2.39\left(3 \mathrm{H}, \mathrm{s}, \mathrm{CH}_{3}\right), 5.63(2 \mathrm{H}$, bs, $\left.\mathrm{NH}_{2}\right), 6.67$ (2H, m, pyrrole H), $7.16(1 \mathrm{H}, \mathrm{m}, \mathrm{ArH}), 7.25(1 \mathrm{H}, \mathrm{dt}, J$ 8.2, 1.5, ArH), $7.58(1 \mathrm{H}$, m, ArH), 7.82 (1H, dd, J 8.2, 1.5, ArH), 9.84 (1H, bs, NH); ${ }^{13} \mathrm{C}-\mathrm{NMR}\left(75 \mathrm{MHz}, \mathrm{CDCl}_{3}\right): \delta 26.7$, 115.4, 116.4, 117.1, 118.3, 127.4, 128.3, 131.9, 132.8, 133.8, 151.1, 186.1, 192.4; m/z (CI): 246 $\left(\mathrm{MNH}_{4}^{+}, 5 \%\right), 229\left(\mathrm{MH}^{+}, 100\right)$.

4-Acetylpyrrol-2-yl 2-(dimethylaminomethylenamino)phenyl ketone (26). To a stirred solution of 2-aminophenyl 4-acetylpyrrol-2-yl ketone 25 (0.8 g, $3.5 \mathrm{mmol})$ in anhydrous DMF (5 ml), t-butoxybis(dimethylamino)methane (Bredereck's reagent) (0.86 ml, $4.2 \mathrm{mmol}, 1.2$ equiv) was added and the reaction mixture was left to stir at rt for $48 \mathrm{~h}$. The reaction mixture was then extracted with $\mathrm{CH}_{2} \mathrm{Cl}_{2}$, the extracts were washed with brine, dried over $\mathrm{MgSO}_{4}$ and concentrated under reduced pressure. Purification by column $\mathrm{SiO}_{2}$ chromatography (50-100\% of EtOAc in hexane $+1 \%$ of Et3N) and subsequent recrystallisation from EtOH afforded 4acetylpyrrol-2-yl 2-(dimethylaminomethylenamino)phenyl ketone $\mathbf{2 6}$ as a white-yellow solid (0.8 g, 82\%), mp 178-179 ${ }^{\circ} \mathrm{C}$; (found C, 67.92; $\mathrm{H}, 5.86 ; \mathrm{N}, 14.46 \% ; \mathrm{C}_{16} \mathrm{H}_{17} \mathrm{~N}_{3} \mathrm{O}_{2}$ requires C, 67.83, H, 6.05, N, 14.83\%); $v_{\max }$ (film): 1098, 1375, 1414, 1432, $1632 \mathrm{~cm}^{-1}$; ${ }^{1} \mathrm{H}-\mathrm{NMR}$ (300 $\mathrm{MHz}_{\mathrm{CDCl}}$ ): $\delta 2.43\left(3 \mathrm{H}, \mathrm{s}, \mathrm{CH}_{3}\right), 2.86\left(3 \mathrm{H}, \mathrm{bs}, \mathrm{NCH}_{3}\right), 2.98\left(3 \mathrm{H}, \mathrm{bs}, \mathrm{NCH}_{3}\right), 6.95$ (1H, d, $J$ 8.1, ArH), 7.10 (1H, t, $J$ apparent t, $J$ 7.9, ArH), 7.16 (1H, s, pyrrole H), 7.42 (1H, apparent t, $J$ 7.9, ArH), 7.47 (1H, s, CH), 7.50 (1H, m, ArH), 7.59 (1H, s, pyrrole H), 11.05 (1H, bs, NH); ${ }^{13} \mathrm{C}-\mathrm{NMR}\left(75 \mathrm{MHz},\left(\mathrm{CD}_{3}\right)_{2} \mathrm{SO}\right): \delta$ 27.6, 118.3, 121.2, 122.6, 127.1, 127.6, 129.7, 131.9, 132.0, 133.4, 134.1, 153.7, 186.9, 194.0; m/z (CI): 284 ( $\left.\mathrm{MH}^{+}, 70 \%\right), 110$ (37), 74 (100).

6-Acetyl-1-(2-(dimethylaminomethylenamino)phenyl)-3H-pyrrolizine (27). To a stirred suspension of 4-acetylpyrrol-2-yl 2-(dimethylaminomethylenamino)phenyl ketone 26 (0.65 g, $2.29 \mathrm{mmol}$ ) and vinyltriphenylphosphonium bromide (0.82 g, $2.29 \mathrm{mmol}, 1$ equiv) in anhydrous THF (50 ml) at $-10{ }^{\circ} \mathrm{C}, \mathrm{NaH}(0.124 \mathrm{~g}$ of a $60 \%$ dispersion in mineral oil, $3.1 \mathrm{mmol}$, $1.35 \mathrm{equiv})$ was added. After $20 \mathrm{~min}$, the reaction mixture was poured into cold water, extracted with $\mathrm{Et}_{2} \mathrm{O}$, the extracts were washed with brine, dried over $\mathrm{MgSO}_{4}$ and concentrated under reduced pressure. Column $\mathrm{SiO}_{2}$ chromatography (25-50\% of AcOEt in hexane $+1 \%$ of $^{2} t_{3} \mathrm{~N}$ ) yielded 6acetyl-1-(2-(dimethylaminomethylenamino)phenyl)-3H-pyrrolizine 27 as a clear yellow oil (0.217 g, 32\%); (found $\mathrm{M}^{+}$, 293.1577. $\mathrm{C}_{18} \mathrm{H}_{19} \mathrm{~N}_{3} \mathrm{O}$ requires $M, 293.1578$ ); $v_{\max }$ (film): 759, 1097, 1182, 1207, 1448, 1287, 1372, 1436, 1478, 1504, 1589, $1632 \mathrm{~cm}^{-1}$; ${ }^{1} \mathrm{H}-\mathrm{NMR}$ (300 MHz, $\left.\mathrm{CDCl}_{3}\right): \delta 2.45\left(3 \mathrm{H}, \mathrm{s}, \mathrm{CH}_{3}\right), 3.05\left(6 \mathrm{H}, \mathrm{s}, \mathrm{N}\left(\mathrm{CH}_{3}\right)_{2}\right), 4.66\left(2 \mathrm{H}, \mathrm{bs}, \mathrm{CH}_{2}\right), 6.56(1 \mathrm{H}, \mathrm{bs}, \mathrm{CH}), 6.87$ (1H, s, H pyrrole), 6.90 (1H, m, ArH), 7.14 (1H, apt, J 7.5, ArH), 7.28 (1H, m, ArH), $7.48(1 \mathrm{H}$, s, NCHN), 7.59 (1H, s, H pyrrole), 7.74 (2H, apd, J 7.5, ArH); ${ }^{1} \mathrm{H}-\mathrm{NMR}\left(300 \mathrm{MHz},\left(\mathrm{CD}_{3}\right)_{2} \mathrm{CO}\right)$ : 


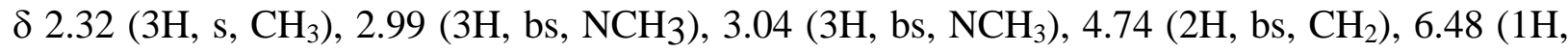
bs, CH), 6.89 (1H, t, J 7.3, ArH), 7.05 (1H, m, pyrrole H), 7.07 (1H, s, NCHN), 7.22 (1H, t, $J$ 7.3, ArH), 7.33 (1H, t, J 7.3, ArH), 7.58 (1H, s, pyrrole H), $7.73\left(2 \mathrm{H}, \mathrm{d}, J\right.$ 7.3, ArH); ${ }^{13} \mathrm{C}-\mathrm{NMR}$ (75 MHz, $\left.\left(\mathrm{CD}_{3}\right)_{2} \mathrm{CO}\right): \delta$ 26.4, 27.2, 52.6, 99.1, 117.8, 120.7, 121.0, 122.2, 122.7, 123.0, 126.7, 129.1, 129.4, 130.5, 134.7, 153.6, 192.6; m/z (CI): 294 ( $\left.\mathrm{MH}^{+}, 100 \%\right)$.

6-Acetyl-1-(2-(dimethylaminomethylenamino)phenyl)-2,3-dihydro-1H-pyrrolizine (28). To a stirred solution of 6-acetyl-2-dimethylaminomethylenamino)phenylpyrrolizine 27 (0.18 g, $0.61 \mathrm{mmol}, 1$ equiv) in $\mathrm{MeOH}(3 \mathrm{ml})$ was added $\mathrm{Pd} / \mathrm{C}(0.018 \mathrm{~g}, 10 \%$ by wt). The resulting mixture was purged three times with $\mathrm{H}_{2}$, and left under a $\mathrm{H}_{2}$ atmosphere overnight. The mixture was then purged with $\mathrm{N}_{2}$, passed through a pad of celite, and evaporated to dryness. Column $\mathrm{SiO}_{2}$ chromatography (50-100\% of EtOAc in hexane, 1\% $\left.\mathrm{Et}_{3} \mathrm{~N}\right)$ yielded 6-acetyl-1-(2(dimethylaminomethylenamino)phenyl)-2,3-dihydro-1H-pyrrolizine $\mathbf{2 8}$ as a clear yellow oil (0.071 g, 39\%); (found $\mathrm{M}^{+}, 295.1685 . \mathrm{C}_{18} \mathrm{H}_{21} \mathrm{~N}_{3} \mathrm{O}$ requires $M, 295.1685$ ); $v_{\max }$ (film): 1100, 1207, 1370, 1435, 1589, 1693, $3258 \mathrm{~cm}^{-1} ;{ }^{1} \mathrm{H}-\mathrm{NMR}$ (300 MHz, $\left.\left(\mathrm{CD}_{3}\right)_{2} \mathrm{CO}\right): \delta 2.28\left(3 \mathrm{H}, \mathrm{s}, \mathrm{CH}_{3}\right)$, $2.36(1 \mathrm{H}, \mathrm{m}, \mathrm{CHH}), 2.93(1 \mathrm{H}, \mathrm{m}, \mathrm{CHH}), 3.04\left(6 \mathrm{H}, \mathrm{s}, \mathrm{N}\left(\mathrm{CH}_{3}\right)_{2}\right), 4.03(1 \mathrm{H}, \mathrm{m}, \mathrm{CHH}), 4.13(1 \mathrm{H}$, m, CHH), 4.87 (1H, t, J 7.7, CH), 6.07 (1H, s, H pyrrole), 6.86 (2H, m, ArH), 7.02 (1H, d, J 7.6, ArH), 7.10 (1H, dt, $J$ 7.6, 1.3, ArH), 7.41 (1H, s, pyrrole H), $7.62(1 \mathrm{H}, \mathrm{s}, \mathrm{NCHN}) ;{ }^{13} \mathrm{C}-\mathrm{NMR}(75$ $\left.\mathrm{MHz},\left(\mathrm{CD}_{3}\right)_{2} \mathrm{CO}\right): \delta$ 25.8, 28.2, 36.8, 38.2, 45.4, 99.7, 118.3, 119.3, 121.9, 126.2, 127.1, 127.2, 135.8, 141.5, 150.0, 152.4, 191.2 ; m/z (CI): 296 ( $\left.\mathrm{MH}^{+}, 100 \%\right), 280$ (5), 149 (2).

1-(2-Aminophenyl)-6-ethyl-2,3-dihydro-1H-pyrrolizine 30 and 1-(2-(dimethylaminomethyl enamino)phenyl)-6-ethyl-2,3-dihydro- $1 \mathrm{H}$-pyrrolizine (29). To a stirred suspension of $\mathrm{AlCl}_{3}$ (0.067 g, 0.51 mmol, 3 equiv) in $\mathrm{CH}_{2} \mathrm{Cl}_{2}(5 \mathrm{ml})$ at rt was added $t-\mathrm{BuNH}_{2} \mathrm{BH}_{3}(0.088 \mathrm{~g}$, $1.02 \mathrm{mmol}, 6$ equiv). The mixture was stirred for $30 \mathrm{~min}$ and then a solution of 6-acetyl-2dimethylaminomethylenamino)phenyl-2,3-dihydro- $1 H$-pyrrolizine 25 (0.05 g, $0.17 \mathrm{mmol}, 1$ equiv) in $\mathrm{CH}_{2} \mathrm{Cl}_{2}(2 \mathrm{ml})$ was added. After $2 \mathrm{~h}$ the reaction was poured into ice and extracted with $\mathrm{CH}_{2} \mathrm{Cl}_{2}$. The combined organic extracts were washed with sat. aq. $\mathrm{NaHCO}_{3}$ and brine, dried over $\mathrm{MgSO}_{4}$ and concentrated under reduced pressure. Column $\mathrm{SiO}_{2}$ chromatography (10\% of EtOAc in hexane) provided firstly 1-(2-aminophenyl)-6-ethyl-2,3-dihydro-1H-pyrrolizine 30 (0.015 g, 40\%) and secondly 1-(2-(dimethylaminomethylenamino)phenyl)-6-ethyl-2,3-dihydro1H-pyrrolizine 29 (0.006 g, 12\%), both as clear oils; spectroscopic data for 30: (found $\mathrm{M}^{+}$, 226.1470. $\mathrm{C}_{15} \mathrm{H}_{18} \mathrm{~N}_{2}$ requires $\left.M, 226.1470\right) ;{ }^{1} \mathrm{H}-\mathrm{NMR}$ (300 MHz, $\left.\left(\mathrm{CD}_{3}\right)_{2} \mathrm{CO}\right): \delta 1.14$ (3H, t, $J$ 7.5, $\left.\mathrm{CH}_{3}\right), 2.28(1 \mathrm{H}, \mathrm{m}, \mathrm{CHH}), 2.44\left(2 \mathrm{H}, \mathrm{q}, J \mathrm{7.5}, \mathrm{CH}_{2}\right), 2.88(1 \mathrm{H}, \mathrm{m}, \mathrm{CHH}), 3.86(1 \mathrm{H}, \mathrm{m}$, $\mathrm{CHH}), 3.95$ (1H, m, CHH), 4.34 (1H, t, J 7.3, CH), 4.36 (2H, bs, $\mathrm{NH}_{2}$ ), 5.80 (1H, s, pyrrole H), 6.45 (1H, s, pyrrole H), 6.53 (1H, dt, $J$ 7.4, 1.1, ArH), 6.70 (1H, dd, J 7.9, 1.1, ArH), 6.90 (2H, m, ArH); ${ }^{13} \mathrm{C}-\mathrm{NMR}\left(75 \mathrm{MHz},\left(\mathrm{CD}_{3}\right)_{2} \mathrm{CO}\right): \delta 15.5,20.5,36.0,39.6,46.3,100.4,111.4,116.3$, 117.9, 123.6, 125.3, 126.5, 127.2, 127.9, 139.1; m/z (CI): $227\left(\mathrm{MH}^{+}, 100 \%\right), 197$ (1), 170 (1); spectroscopic data for 29: (found $\mathrm{M}^{+}$, $281.1896 \mathrm{C}_{18} \mathrm{H}_{23} \mathrm{~N}_{3}$ requires $M$, 281.1892); ${ }^{1} \mathrm{H}-\mathrm{NMR}$ (300 $\left.\mathrm{MHz},\left(\mathrm{CD}_{3}\right)_{2} \mathrm{CO}\right): \delta 1.13\left(3 \mathrm{H}, \mathrm{t}, J\right.$ 7.6, $\left.\mathrm{CH}_{3}\right), 2.15(1 \mathrm{H}, \mathrm{m}, \mathrm{CHH}), 2.42\left(2 \mathrm{H}, \mathrm{q}, J\right.$ 7.6, $\left.\mathrm{CH}_{2}\right), 2.85$ (1H, m, CHH), 3.01 (6H, bs, $\left.\mathrm{N}\left(\mathrm{CH}_{3}\right)_{2}\right), 3.83(1 \mathrm{H}, \mathrm{m}, \mathrm{CHH}), 3.94(1 \mathrm{H}, \mathrm{m}, \mathrm{CHH}), 4.78(1 \mathrm{H}, \mathrm{t}, J$ 7.3, CH), 5.48 (1H, s, pyrrole H), $6.41(1 \mathrm{H}$, s, pyrrole H), $6.77(1 \mathrm{H}$, dd, J 7.6, 1.2, ArH), 6.83 (1H, dt, J 7.6, 1.2, ArH), 7.03 (2H, m, ArH), 7.58 (1H, s, NCHN); m/z (CI): $282\left(\mathrm{MH}^{+}, 100 \%\right)$. 
6-Ethyl-2,3,4,7a-tetrahydro-7a-hydroxy-1H-1-phenylpyrrolizin-5-one (31). To a stirred solution of 6-ethyl-2,3-dihydro-1-phenyl-1H-pyrrolizine 19 (0.07 g, $0.33 \mathrm{mmol})$ in $\mathrm{CH}_{2} \mathrm{Cl}_{2}$ $(20 \mathrm{ml})$ at $-78{ }^{\circ} \mathrm{C}$ was added methylene blue $\left(3.3 \mathrm{ml}\right.$ of a $0.001 \mathrm{M}$ solution in $\mathrm{CH}_{2} \mathrm{Cl}_{2}$, $0.0033 \mathrm{mmol}, 0.01$ equiv). The mixture was maintained at $-78{ }^{\circ} \mathrm{C}$ and irradiated for 90 min with a Hg medium pressure lamp. The solvent was evaporated at less than $45^{\circ} \mathrm{C}$, and the residue purified by column $\mathrm{SiO}_{2}$ chromatography (5-25-50\% of EtOAc in hexane) to give 31 as a yellow oil (0.019 g, 24\%); (found $\mathrm{M}^{+}$, 243.1262. $\mathrm{C}_{15} \mathrm{H}_{17} \mathrm{NO}_{2}$ requires $\mathrm{M}, 243.1259$ ); $v_{\max }$ (film): 1069, 1409, 1446, 1706, 2928, 3191-3400 cm ${ }^{-1}$; ${ }^{1} \mathrm{H}-\mathrm{NMR}$ (300 MHz, CD ${ }_{3} \mathrm{CO}$ ): $\delta 1.22$ (3H, t, J 7.5, $\left.\mathrm{CH}_{3}\right), 2.01$ (2H, m, CHH), $2.41\left(2 \mathrm{H}, \mathrm{q}, J\right.$ 7.5, $\left.\mathrm{CH}_{2}\right), 2.87(1 \mathrm{H}, \mathrm{m}, \mathrm{CHH}), 3.50(1 \mathrm{H}, \mathrm{m}, \mathrm{CHH})$, 3.82 (1H, t, J 7.7, CH), 7.30 (1H, s, pyrrole H), 7.35 (2H, m, ArH), 7.47 (2H, apd, J 7.5, ArH), 7.52 (1H, apt, $J$ 7.5, ArH); ${ }^{13} \mathrm{C}-\mathrm{NMR}$ (75 MHz, $\left.\mathrm{CD}_{3} \mathrm{CO}\right): \delta 12.5,20.4,36.7,39.7,68.0,120.7$, 127.8, 128.7, 129.6, 135.1, 140.0, 158.8, 188.6; m/z (CI): 243 ( $\left.\mathrm{MH}^{+}, 14 \%\right), 226$ (100).

\section{Acknowledgements}

We thank the UK EPSRC for the project studentship (CCL) that provided the funding for this work.

\section{References}

1. The Chemistry of Heterocyclic Compounds, Vol 25, Indoles, Part 4, Saxton, J. E., Ed. Wiley-Interscience: New York, 1983 and Supplement to Part 4, Saxton, J. E., Ed.;WileyInterscience: New York, 1994.

2. Chapters VI in reference 1; Alvarez, M.; Joule, J. A. The Alkaloids 2001, 57, 235.

3. Chapters VIII in reference 1; Saxton, J. E. The Alkaloids 1998, 51, 2.

4. Chapters V in reference 1.

5. Ramírez, A.; García-Rubio, S. Curr. Med. Chem. 2003, 10, 1981.

6. (a) Joule, J. A.; Smith, G. F. J. Chem. Soc.1962, 312. (b) Britten, A. Z.; Joule, J. A.; Smith, G. F. Chem. Ind. 1963, 1120. (c) Britten, A. Z.; Joule, J. A.; Smith, G. F. Tetrahedron 1967, 23, 1971.

7. (a) Olivier-Bever, B. J. Ethnopharmacol. 1982, 5, 1. (b) Neuwinger, H. D. African Ethnobotany. Poisons and Drugs Chapman \& Hall: Weinheim 1996, p 123.

8. Ali, B. H.; Al-Qarawi, A. A.; Bashir, A. K.; Tanira, M. O. Phytother. Res. 2000, 14, 229.

9. Subhadhirasaul, S.; Takayama, H.; Miyabe, Y.; Aimi, N.; Ponglux, D.; Sakai, S.-I. Chem. Pharm. Bull. 1994, 42, 2645.

10. Henry, T. A.; Sharp, T. M. J. Chem. Soc. 1927, 1950.

11. Henry, T. A. J. Chem. Soc. 1932, 2759.

12. (a) Hamilton, J. A.; Hamor, T. A.; Robertson, J. M.; Sim, G. A. Proc. Chem. Soc. 1961, 63. (b) Manohar, H.; Ramasehan, S. Curr. Sci. In. 1961, 30, 5. 
13. Olivier, L.; Lévy, J.; Le Men, J.; Janot, M.-M.; Budzikiewicz, H.; Djerassi, C. Bull. Soc. Chim. Fr. 1965, 868.

14. (a) Iwu, M. M.; Klayman, D. L. J. Ethnopharmacol. 1992, 36, 133. (b) Fayeke, T. O.; Itiola, O. A.; Odeloa, H. A. Phytother. Res. 2000, 14, 368.

15. Leewanich, P.; Tohda, M.; Matsumoto, K.; Subhadhirasakul, S.; Takayama, H.; Aimi, N.; Watanabe, H. Biol. Pharm. Bull. 1996, 19, 394.

16. Lin, S. C.; Lin, C. C.; Lin, H. Y.; Supriyatna, S. C.; Pan, S. L. Am. J. Clin. Med. 1996, 24, 153.

17. (a) Karamajan, P.; Sekar, N.; Mathuram, V.; Govindasamy, S. Biochem. Int. 1991, 25, 491.

(b) Karamajan, P.; Sekar, N.; Govindasamy, S. Med. Sci. Res. 1995, 23, 237.

18. Evans, B. E.; Rittle, K. E.; Bock, M. G.; DiPardo, R. M.; Fredinger, R. M.; Whitter, W. L.; Lundell, G. F.; Veber, D. F.; Anderson, P. S.; Chang, R. S. L.; Lotti, V. J.; Cerino, D.J.; Chen, T. B.; Kling, P. J.; Kunkel, K. A.; Springer, J. P.; Hirshfield, J. J. Med. Chem. 1988, 31, 2235.

19. (a) Dolby, L. J.; Esfandiari, Z. J. Org. Chem. 1972, 37, 43. (b) Dolby, L. J.; Nelson, S. J. J. Org. Chem. 1973, 38, 2882.

20. Wenkert, E.; Guo, M.; Pestchanker, M. J.; Shi, Y. -J.; Vankar, Y. D. J. Org. Chem. 1989, 54, 1166.

21. Smith, G. F. Chem. Ind. 1961, 1120.

22. Koike, T.; Takayama, H.; Sakai, S. Chem. Pharm. Bull. 1991, 39, 1677.

23. Lévy, J.; Sapi, J.; Laronze, J. -Y.; Royer, D.; Toupet, L. Synlett 1992, 601.

24. (a) Bennasar, M.-L.; Zulaica, E.; Ramírez, A.; Bosch, J. J. Org. Chem. 1996, 61, 1239. (b) Bennasar, M.-L.; Zulaica, E.; Ramírez, A.; Bosch, J. Tetrahedron Lett. 1996, 37, 6611. (c) Bennasar, M.-L.; Zulaica, E.; Ramírez, A.; Bosch, J. Heterocycles 1998, 43, 1959. (d) Bennasar, M.-L.; Zulaica, E.; Ramírez, A.; Bosch, J. Tetrahedron 1999, 55, 3117.

25. (a) Lightner, D. A.; Bisacchi, G. S.; Norris, R. D. J. Am. Chem. Soc. 1976, 98, 802. (b) Natsume, M.; Muratake, H. Tetrahedron Lett. 1979, 3477. (c) Muratake, H.; Natsume, M. Tetrahedron Lett. 1989, 30, 5771.

26. Lee, G. C. M.; Holmes, J. M.; Harcourt, D. A.; Garst, M. E. J. Org. Chem. 1992, 57, 3126.

27. (a) Martin, S. F.; Chen, H.-J.; Lynch, V. M. J. Org. Chem. 1993, 58, 2867. (b) Martin, S. F.; Chen, H.-J.; Lynch, V. M. J. Org. Chem. 1995, 60, 276.

28. Mannich, A. Chem. Ber. 1935, 68, 113.

29. Hammen, P. D.; Braisted, A. C.; Northrup, D. L. Synth. Commun. 1991, 21, 2157.

30. Fraser, M. M.; Raphael, R. A. J. Chem. Soc. 1952, 226.

31. Mitchell, D.; Koening, T. M. Synth. Commun. 1995, 25, 1231.

32. Campiani, G.; Nacci, V.; Bechelli, S.; Ciani, S. M.; Garofalo, A.; Fiorini, I.; Wikstrom, A.; Boer, P.; Liao, Y.; Tepper, P.G.; Cagnotto, A.; Mennini, T. J. Med. Chem. 1998, 41, 3763.

33. (a) Gmeiner, P.; Lerche, H. Heterocycles 1990, 31, 9. (b) Gmeiner, P. Heterocycles 1991, 32, 1499.

34. Ghosh, A. K.; Lei, H. J. Org. Chem. 2002, 67, 8783.

35. Nakajima, N.; Horita, K.; Abe, R.; Yonemitsu, O. Tetrahedron Lett. 1988, 29, 4139. 
36. Bourgeois, M. J.; Mountaudon, E.; Maillard, B. Tetrahedron 1993, 49, 2477.

37. Katritzky, A. R.; Fali, C. N.; Li, J. J. Org. Chem. 1997, 62, 4148.

38. Linderman, R. J.; Meyers, A. I. Heterocycles 1983, 20, 1737.

39. Schweizer E. E.; Light, K. K. J. Org. Chem. 1966, 31, 870.

40. Rokach, J.; Hamel, P.; Kakushima, M.; Smith, G. M. Tetrahedron Lett. 1981, 22, 4901.

41. Cadamuro, S.; Degani, I.; Dughera, S.; Fochi, R.; Gatti, A. J. Chem. Soc., Perkin Trans. 1 1993, 273.

42. Ketcha, D. M.; Carpenter, K. P.; Atkinson, S. T.; Rajagopalan, H. R. Synth. Commun. 1990, 20, 1647.

43. Kimbaris, A.; Varvounis, G. Tetrahedron 2000, 56, 9675.

44. Okabe, M.; Sun, R.-C. Tetrahedron 1995, 51, 1861.

45. Knapp, S.; Hale, J. J.; Bastos, M.; Gibson, F. Tetrahedron Lett. 1990, 31, 2109.

46. Angle, S. R.; Fevig, J. M.; Knight, S. D.; Marquis, R. W.; Overman, L. E. J. Am. Chem. Soc. 1993, 115, 3966.

47. Katritzky, A. R.; Li, J.; Malhotra, N. Liebigs Ann. Chem. 1992, 843.

48. Anderson, H. J.; Loader, C. E.; Xun Xu, R.; Le, N.; Gogan, N. J.; McDonald, R.; Edwards, L. G. Can. J. Chem. 1985, 63, 896.

49. Eckroth, D. R.; Squire, R. H. J. Org. Chem. 1971, 36, 224.

50. Fürstner, A.; Ernst, A.; Krause, E.; Ptock, A. Tetrahedron 1996, 52, 7329. 